\title{
Metal-dependent Reactivity Differences for Transients Formed By Flash Photolysis of (PNP)M(CO), M = Co and Rh \\ R. Dale Rimmer ${ }^{\S}$, David C. Grills ${ }^{\dagger *}$, Hongjun Fan ${ }^{\ddagger}$, Peter C. Ford ${ }^{{ }^{\star}}$, and Kenneth G. Caulton ${ }^{\ddagger \star}$ \\ Departments of Chemistry, University of California, Santa Barbaraß; Brookhaven National Laboratory, Upton, NY†; and Indiana University, Bloomington, IN $N^{\ddagger}$. \\ RECEIVED DATE: E-Mail: caulton@indiana.edu
}

Materials. Spectrophotometric grade cyclohexane (Aldrich) was distilled over $\mathrm{CaH}_{2}$ under dinitrogen; and then degassed at reduced pressure and stored in an argon filled (99.998\%) glove box. All gases were purchased from Praxair. Argon (99.998\%) was further purified by passage through a column of Drierite and a chromous silica gel column to remove adventitious water and oxygen. Carbon monoxide $(99.5 \%)$ was used as received.

Instrumentation. Electronic absorption spectra for solutions were collected in a $1.0 \mathrm{~cm}$ path length quartz cell using a Shimadzu UV-2401 PC UV/Vis spectrometer. Infrared specta were recorded using a Matson research series FTIR spectrometer.

Photolysis Solutions. Sample solutions were prepared in an argon atmosphere using standard glovebox techniques. Then, argon was removed by three freeze-pump-thaw and the samples were entrained with the appropriate gas at $25{ }^{\circ} \mathrm{C}$ to achieve the desired partial pressure using a mercury manometer and standard Schlenk techniques.

Flash Photolysis instrumentation. The nanosecond time-resolved optical (TRO) system at UCSB uses a photomultiplier tube (PMT) detector to obtain kinetic traces at a single monitoring wavelength $\left(\lambda_{\text {mon }}\right)$ and has been previously described in detail. ${ }^{1,2}$ The pump source $\left(\lambda_{\text {irr }}\right)$ for all UV/Vis transient absorption studies was a $\sim 10 \mathrm{~ns}$ pulse of $355 \mathrm{~nm}$ radiation obtained from the frequency-tripled output of a Continuum N-61 ND:YAG pulsed laser. The laser power was attenuated to obtain approx 80 $\mathrm{mJ} / \mathrm{pulse}$.

Time-resolved infrared (TRIR) spectra were recorded using the UCSB apparatus for tunable single-frequency detection $\left(2150-1550 \mathrm{~cm}^{-1}\right)$ has been described in detail elsewhere. ${ }^{3}$ In the present case, the excitation source was the third harmonic of a Spectra Physics Quanta-Ray Nd:YAG laser $\left(\lambda_{\text {irr }}=\right.$ $355 \mathrm{~nm}, 80 \mathrm{~mJ} /$ pulse).

Low-Temperature Photolyses. Low-temperature FTIR experiments were carried out using a PFDFT12.5 Pourfill Dewar (R.G. Hansen and Associates) with a sample IR cell built to fit a Matson Research Series FTIR spectrometer. ${ }^{2}$ 


\section{References.}

1. Crane, D.R.; Ford, P.C. J. Am. Chem Soc. 1991, 113, 8510-8516.

2. Bridgewater, J.S.; Netzel, T.L., Schoonover, J.R. Massick, S.M.; Ford, P.C. Inorg. Chem. 2001, 40 1446-1476.

3. (a) McFarlane, K. L. Ph.D. Dissertation, University of California, Santa Barbara, 1996. (b) DiBenedetto, J.; Ryba, D. W.; Ford, P. C. Inorg. Chem. 1989, 28, 3503-3507. (c) Ford, P. C.; DiBenedetto, J. A.; Ryba, D. W.; Belt, S. T. SPIE Proc. 1992, 1636, 9-16. (d) Boese, W. T.; Ford, P. C. J. Am. Chem. Soc. 1995, 117, 8381-8391.

\section{Flash photolysis of $(\mathrm{PNP}) \mathrm{Co}(\mathrm{CO})\left(\mathrm{PNP}=\left[\left({ }^{\mathrm{t}} \mathrm{Bu}_{2} \mathrm{PCH}_{2} \mathrm{SiMe}_{2}\right)_{2} \mathrm{~N}\right]^{1-}\right)$ :}

Figure 1 shows the electronic absorption spectrum of a $3.5 \times 10^{-4} \mathrm{M}$ solution of (PNP)Co(CO) (1) in cyclohexane. The spectrum of $\mathbf{1}$ is compared to that of a cyclohexane solution of the carbonylfree species (PNP)Co in Figure 2.

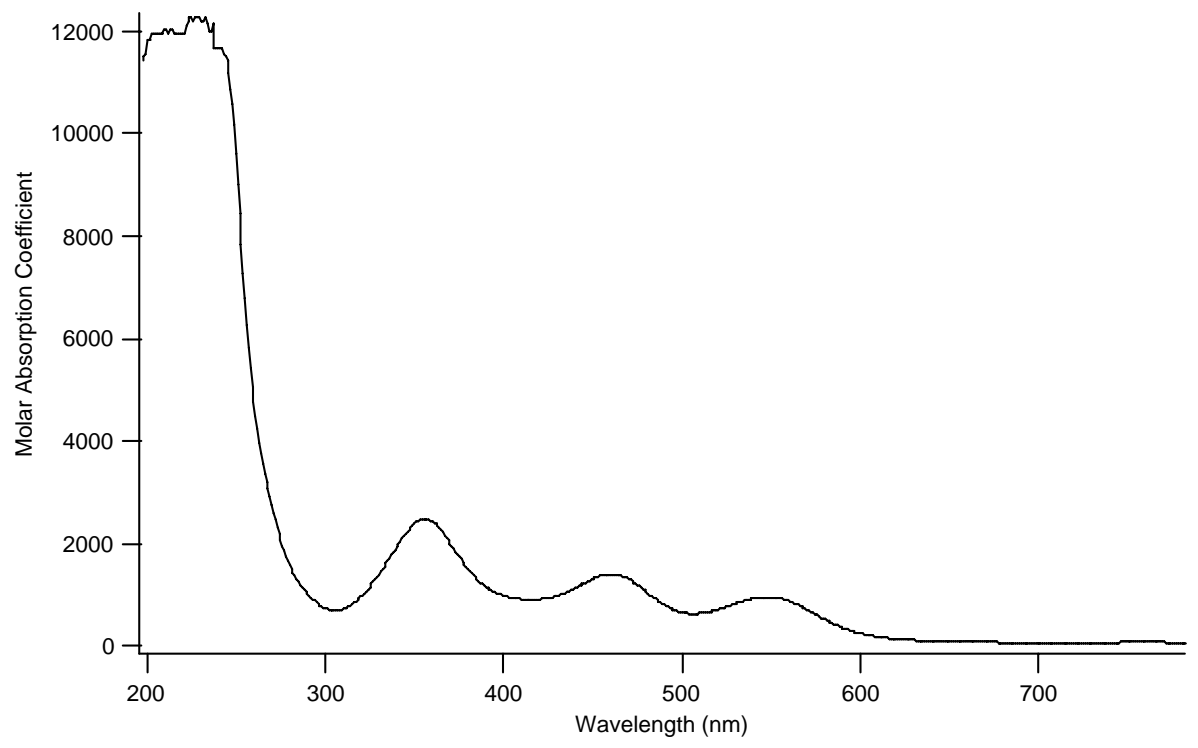

Figure 1. Electronic absorption spectrum of a $3.5 \times 10^{-4} \mathrm{M}(\mathrm{PNP}) \mathrm{Co}(\mathrm{CO})$ cyclohexane solution. 


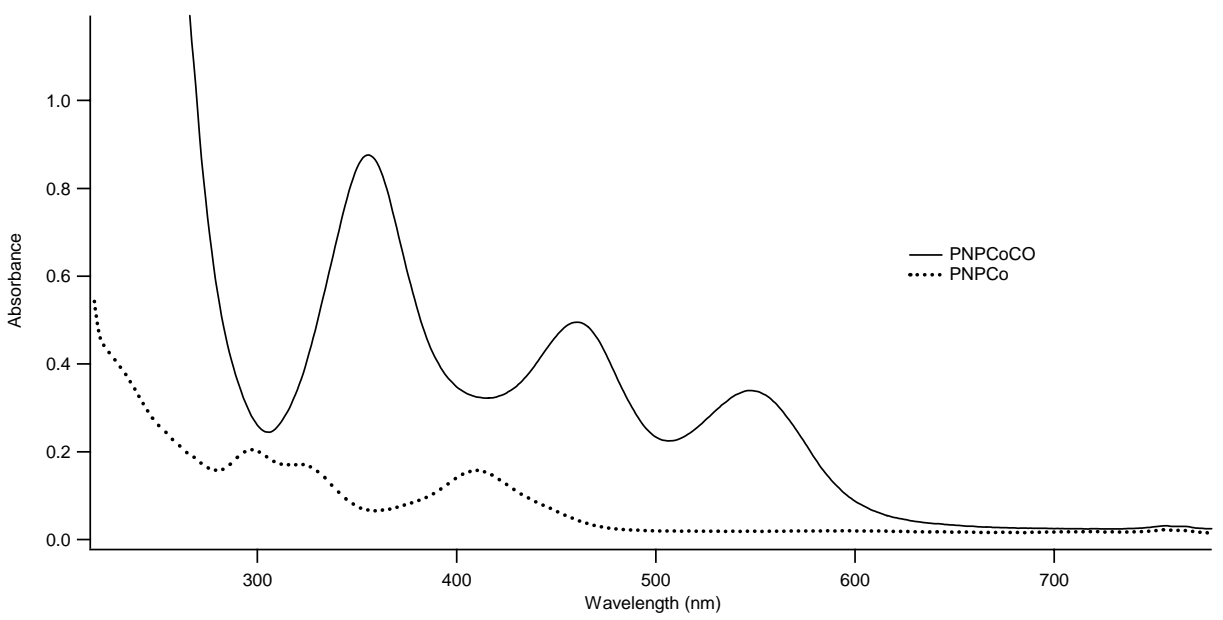

Figure 2. Electronic absorption spectra of ( $\mathrm{PNP}) \mathrm{Co}(\mathrm{CO})$ and $(\mathrm{PNP}) \mathrm{Co}$ recorded in cyclohexane.

Flash photolysis at $355 \mathrm{~nm}$ of the cyclohexane solution of $\mathbf{1}$ under one atmosphere $\mathrm{CO}$ with time resolved optical detection (TRO), generates a transient which absorbs more at $420 \mathrm{~nm}$ (transient absorbance) and less at $460 \mathrm{~nm}$ and $547 \mathrm{~nm}$ (transient bleach). These spectral changes are consistent with $\mathrm{CO}$ dissociation to generate the parent complex (PNP)Co. At all three wavelengths, the transient spectral changes showed single exponential time evolution decay back to the original absorbance over $\sim 5$ half-lives. The three independent rate constants are statistically equivalent at $k_{o b s}=7.3( \pm 1.5) \times 10^{3}$ $\mathrm{s}^{-1}$. A plot of the observed rate constant $\left(k_{o b s}\right)$ at varying CO concentrations (Figure 3 ) is linear with a near-zero intercept consistent with a first-order dependence of [CO]. This establishes an overall rate law $k_{C O}[\mathrm{Co}][\mathrm{CO}]$ with $k_{C O}=9.3( \pm 0.2) \times 10^{5} \mathrm{M}^{-1} \mathrm{~s}^{-1}$, consistent with the reversible photodissociation of $\mathrm{CO}$ illustrated in eq. 1.

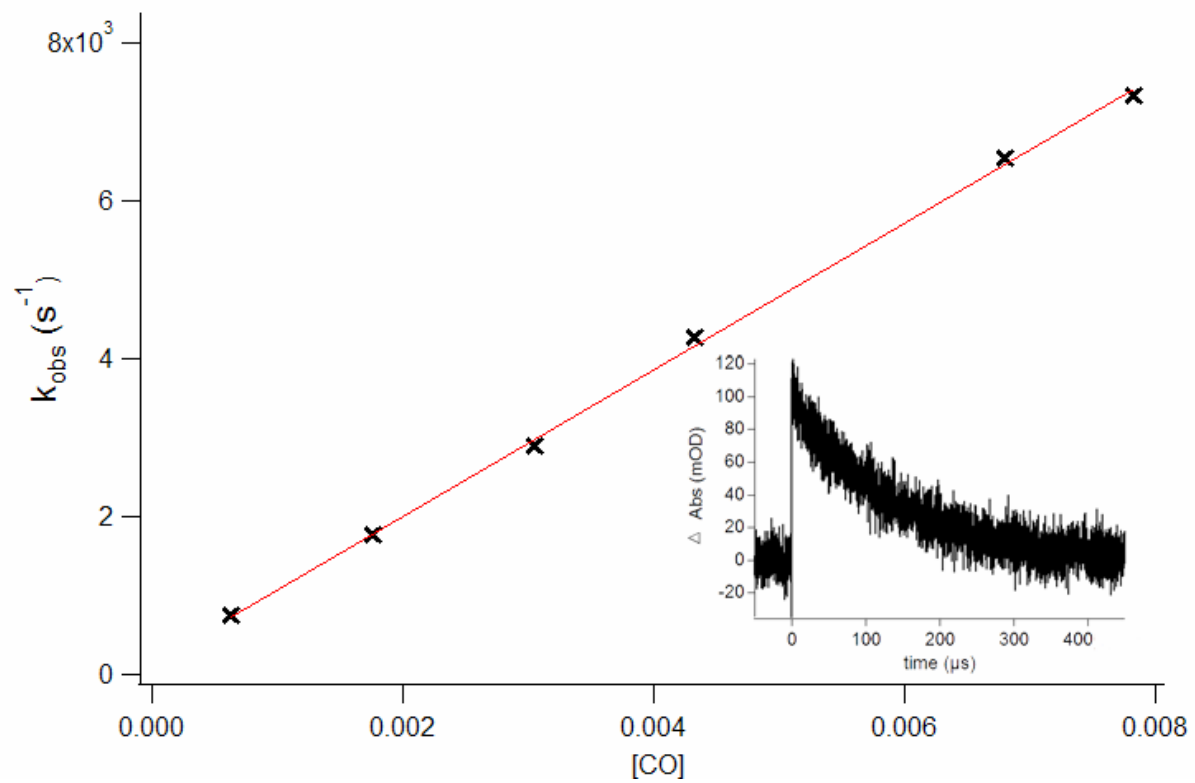


Figure 3. Temporal decay of the transient formed after laser flash photolysis of (PNP)Co(CO) in cyclohexane vs [CO]. The slope of the calculated best fit line depicted is the second-order rate constant $k_{C O}=9.3( \pm 0.2) \times 10^{5} \mathrm{M}^{-1} \mathrm{~s}^{-1}$. Insert: Transient absorbance trace at $420 \mathrm{~nm}$ upon flash photolysis. $\left(\mathrm{T}=25^{\circ} \mathrm{C}\right)$

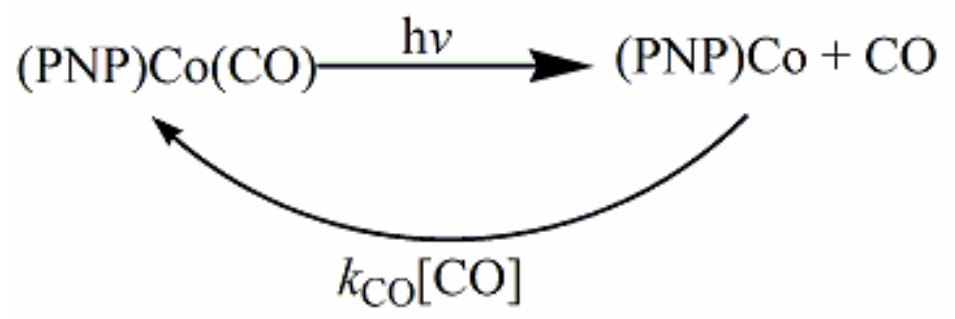

Analogous time resolved infrared studies (TRIR) demonstrated a bleach of the $v_{\mathrm{CO}}$ band of (PNP)Co(CO) at $1885 \mathrm{~cm}^{-1}$ (Figure 4) upon flash photolysis in cyclohexane solution (inset, Figure 5). The recovery of this absorbance is exponential in the presence of excess $\mathrm{CO}$, and the $k_{o b s}$ values so determined are first order in [CO] as predicted by eq. 1 . The slope of the linear $k_{o b s}$ vs. [CO] plot (Figure 5) gives the second order rate constant $k_{C O}=8.0( \pm 1.5) \times 10^{5} \mathrm{M}^{-1} \mathrm{~s}^{-1}$ in agreement (within experimental uncertainty) with the second order rate constant for transient decay measured using TRO techniques (see above).

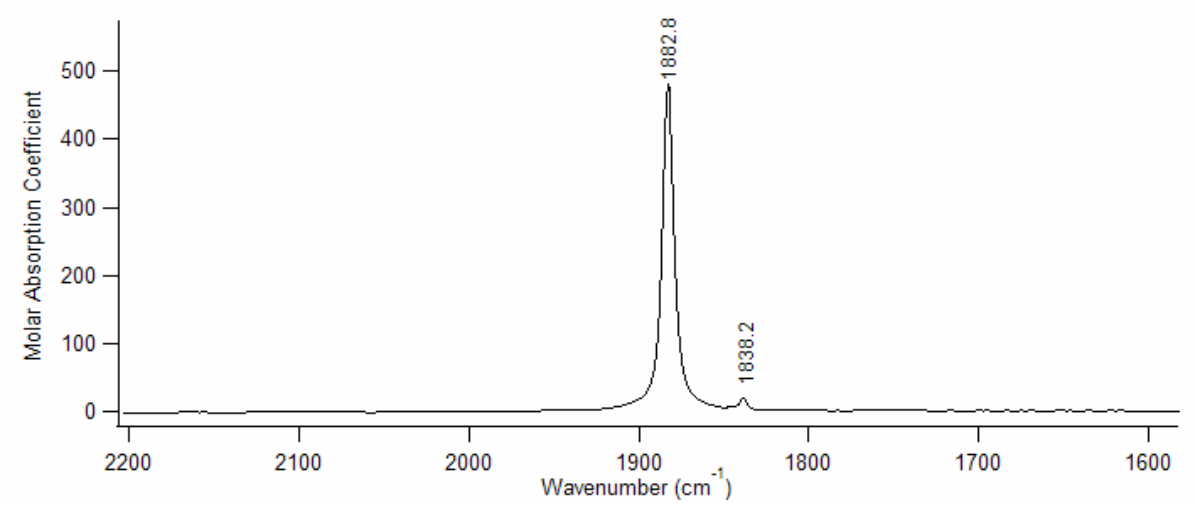

Figure 4. FTIR spectrum of (PNP) $\mathrm{Co}(\mathrm{CO})$ in cyclohexane. 


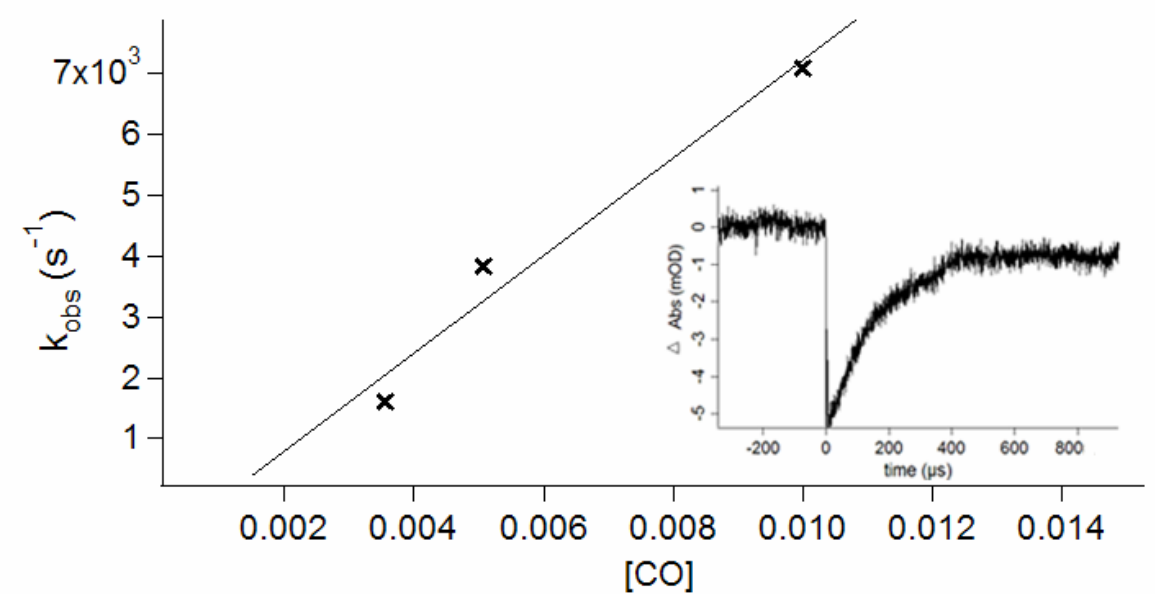

Figure 5. Reformation of (PNP)Co(CO) after laser flash photolysis in cyclohexane vs [CO]. The slope of the calculated best fit line depicted is the second-order rate constant $k_{C O}=8.0( \pm$ 1.5) $\times 10^{5} \mathrm{M}^{-1} \mathrm{~s}^{-1}$. Insert: Transient bleach trace at $1883 \mathrm{~cm}^{-1}$ upon flash photolysis. $\left(\mathrm{T}=25^{\circ} \mathrm{C}\right)$ 


\section{Flash photolysis of (PNP)Rh(CO):}

The electronic absorption spectrum of a $2.4 \times 10^{-4} \mathrm{M}$ solution of (PNP)Rh(CO) (2) in cyclohexane is shown in Figure 6. Flash photolysis at $355 \mathrm{~nm}$ of this solution under one atmosphere $\mathrm{CO}$ generated the point-by-point TRO spectrum shown in Figure 7 with a transient absorbance increase at $470 \mathrm{~nm}$ and a transient bleach at $360 \mathrm{~nm}$.

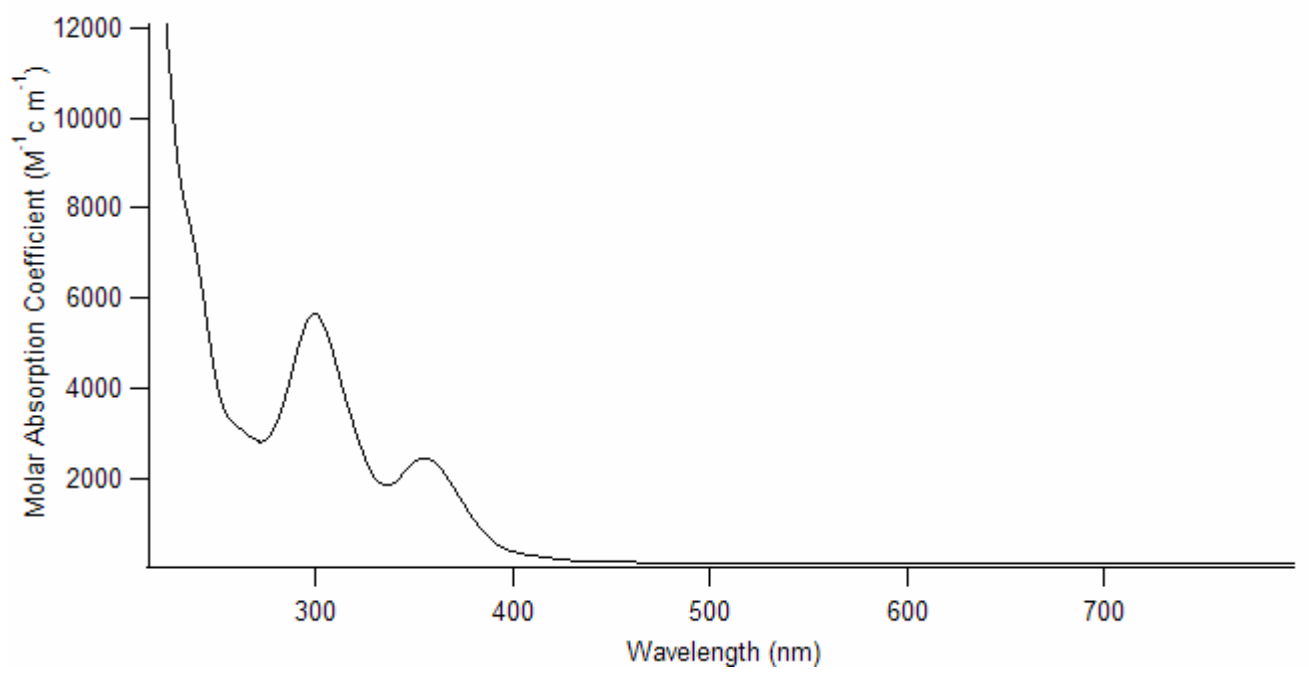

Figure 6. Electronic absorption spectrum of (PNP)Rh(CO) in cyclohexane.

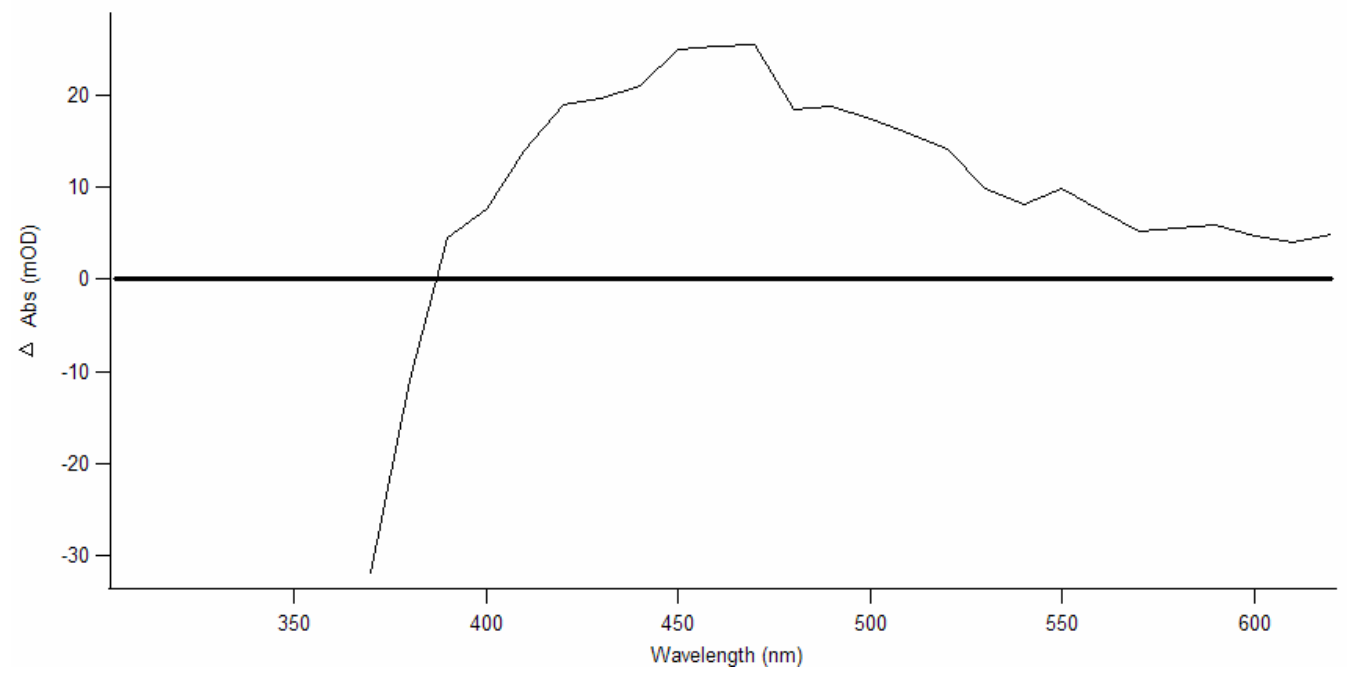

Figure 7. Point by point transient difference spectrum recorded $20 \mu \mathrm{s}$ after $355 \mathrm{~nm}$ flash photolysis of (PNP)Rh(CO) $\left(2.58 \times 10^{-4} \mathrm{M}\right)$ in cyclohexane.

The decay of these transient spectral changes show single exponential time evolution over $\sim 5$ half-lives with an observed rate constant $k_{o b s}=5.7( \pm 0.2) \times 10^{4} \mathrm{~s}^{-1}$. A plot of the rate constant $\left(k_{o b s}\right)$ at 
varying CO concentration (Figure 8) is linear and consistent with a first-order dependence of [CO]. This establishes the overall rate law $\left(k_{1}+k_{C O}[\mathrm{CO}]\right)[\mathrm{Rh}]$ with $k_{C O}=6.3( \pm 1.0) \times 10^{6} \mathrm{M}^{-1} \mathrm{~s}^{-1}$. Although, a non zero intercept of $k_{1}=1.5( \pm 0.4) \times 10^{2} \mathrm{~s}^{-1}$ was seen, the experimental scatter in the $k_{o b s}$ values makes this value too uncertain to have confidence that there really is a CO-independent process occurring.

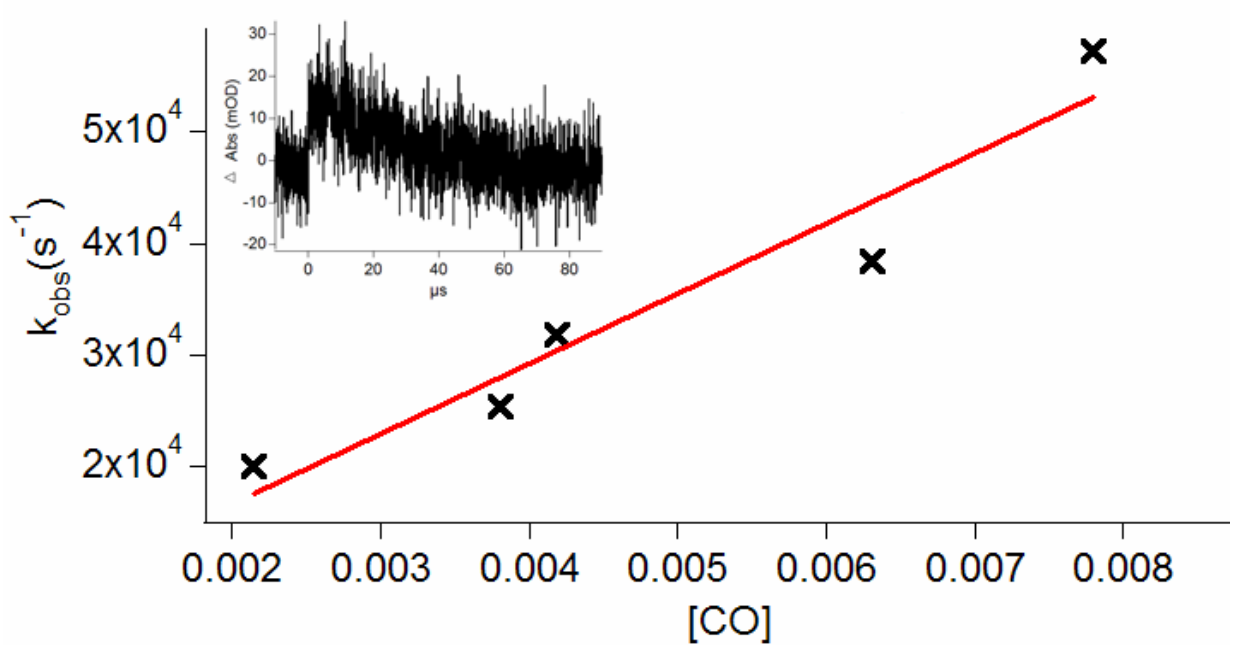

Figure 8. Temporal decay of the transient formed after laser flash photolysis of (PNP)Rh(CO) in cyclohexane vs. [CO] $\left(\lambda_{\text {mon }}=470 \mathrm{~nm}, \mathrm{~T}=25{ }^{\circ} \mathrm{C}\right)$. The slope of the calculated best fit line depicted is the second-order rate constant. Insert: Transient absorbance trace at $470 \mathrm{~nm}$ upon flash photolysis.

The temporal behavior of the transient bleach of the (PNP)Rh(CO) band at $360 \mathrm{~nm}$ upon $355 \mathrm{~nm}$ flash photolysis is quite different. This recovers with an observed rate constant of $3.3( \pm 0.3) \times 10^{1} \mathrm{~s}^{-1}$ for a $2.4 \times 10^{-4} \mathrm{M}$ solution under one atmosphere of $\mathrm{CO}$ (Figure 9), but the recovery rate of this band was unaffected by changing the $\mathrm{CO}$ concentration. Thus, one can conclude that the flash photolysis of (PNP)Rh(CO) leads to the formation of at least two different transients, one of which is analogous to that seen for the cobalt analog and formed by simple $\mathrm{CO}$ dissociation. The other transient species is considerably different in character. 


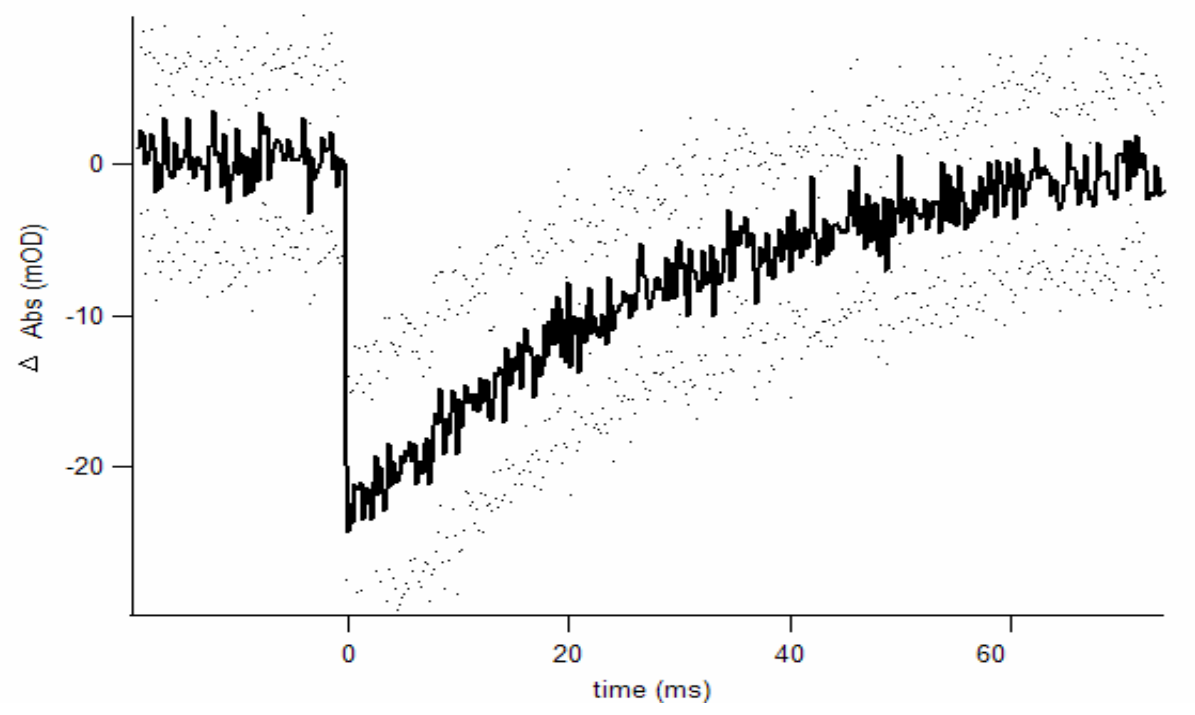

Figure 9. Temporal transient bleach trace after flash photolysis for $(\mathrm{PNP}) \mathrm{Rh}(\mathrm{CO})$ in cyclohexane and one atmosphere $\mathrm{CO}\left(\lambda_{\mathrm{mon}}=360 \mathrm{~nm}, \mathrm{~T}=25^{\circ} \mathrm{C}\right)$.

The FTIR spectrum of (PNP) Rh(CO) (CO region) is shown in Figure 10. Flash photolysis with TRIR detection leads to a transient bleach of the $1931 \mathrm{~cm}^{-1}$ band of 2 , which decayed back to baseline (Figure 11). The observed rate constant for this decay was $3.9( \pm 0.2) \times 10^{1} \mathrm{~s}^{-}$ ${ }^{1}$ and, like the behavior of the transient bleach seen in the TRO experiments, this rate constant was independent of the $\mathrm{CO}$ concentration.

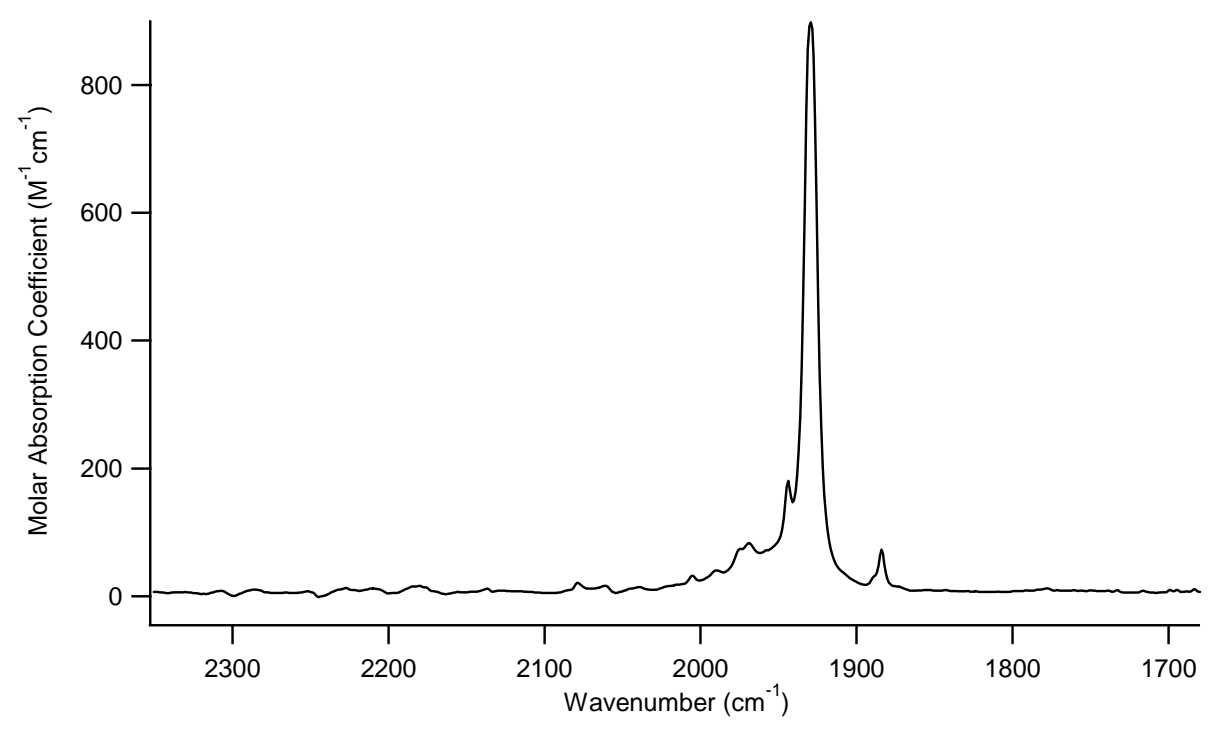

Figure 10. Infrared absorption spectrum (CO region) of (PNP)Rh in cyclohexane. 


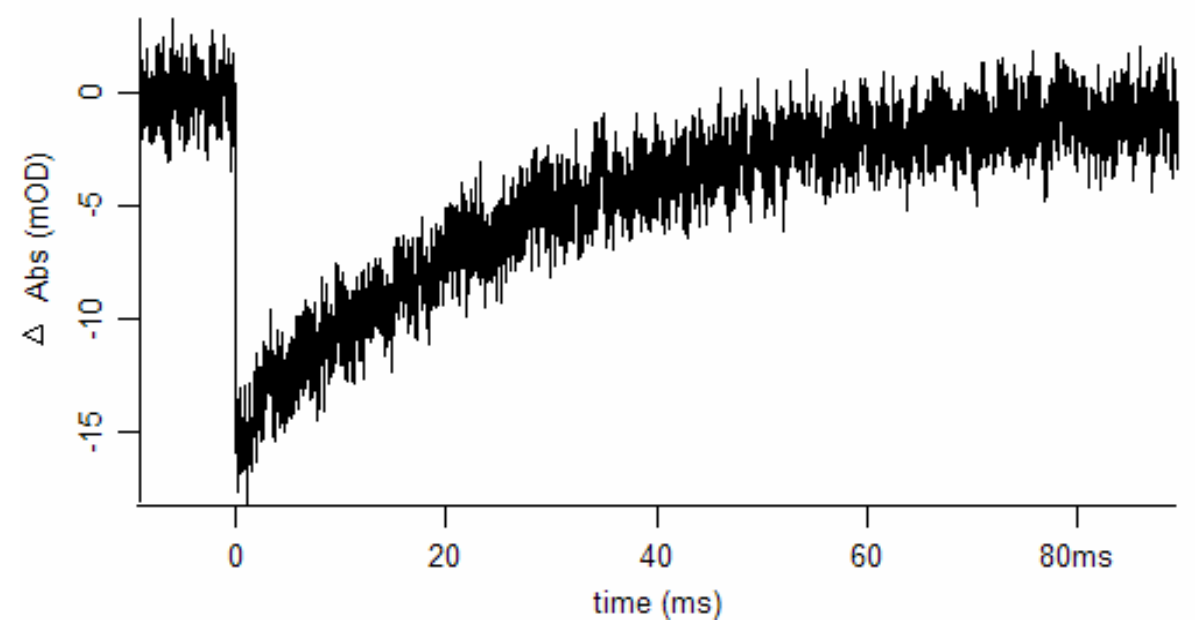

Figure 11. Temporal transient bleach at $1931 \mathrm{~cm}^{-1}$ upon flash photolysis for ( $\left.\mathrm{PNP}\right) \mathrm{Rh}(\mathrm{CO})$ in cyclohexane and one atmosphere CO.

The IR spectral changes accompanying the continuous photolysis $(366 \mathrm{~nm}$ interference filter isolated) of a $\mathrm{N}_{2}$ saturated (PNP)Rh(CO) $\left(1.7 \times 10^{-2} \mathrm{M}\right)$ solution in cyclohexane are shown in Figure 12. The band at $1931 \mathrm{~cm}^{-1}$ was observed to decrease in intensity and a band increased in intensity at $2090 \mathrm{~cm}^{-1}$.

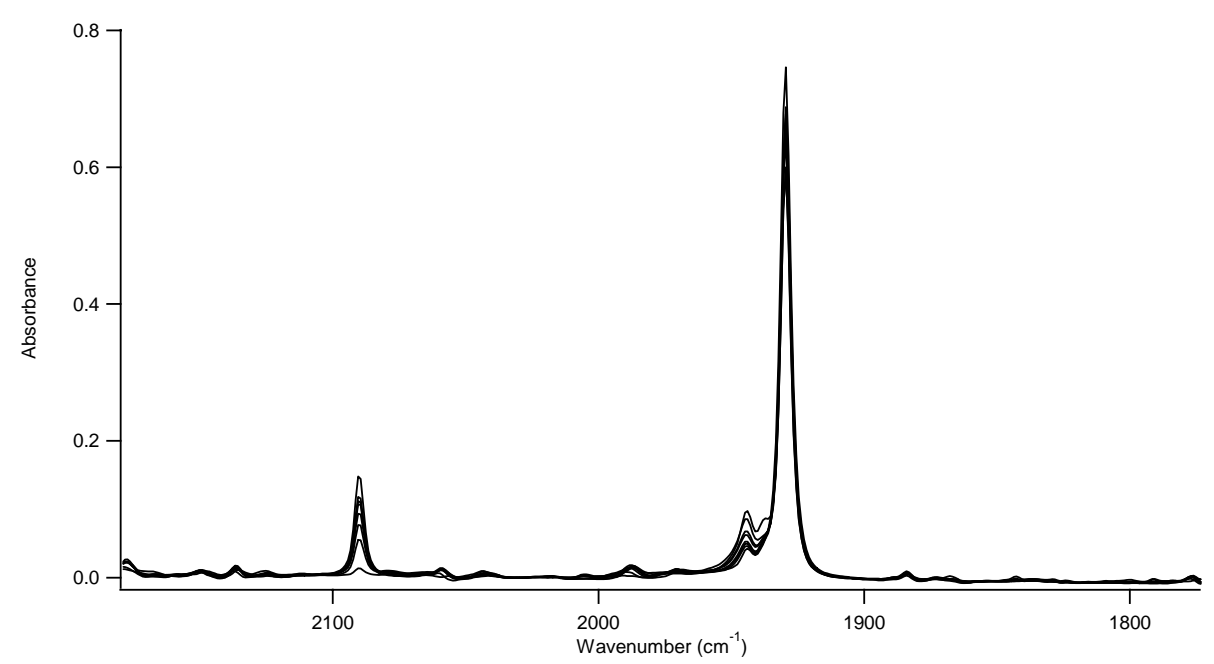

Figure 12. The IR spectral changes accompanying $366 \mathrm{~nm} \mathrm{CW}$ photolysis of a $1.7 \times 10^{-2} \mathrm{M}$ (PNP) $\mathrm{Rh}(\mathrm{CO})$ in cyclohexane saturated with $\mathrm{N}_{2}$. The band at $1931 \mathrm{~cm}^{-1}$ decreased in intensity and the band at 2090 increases in intensity. 


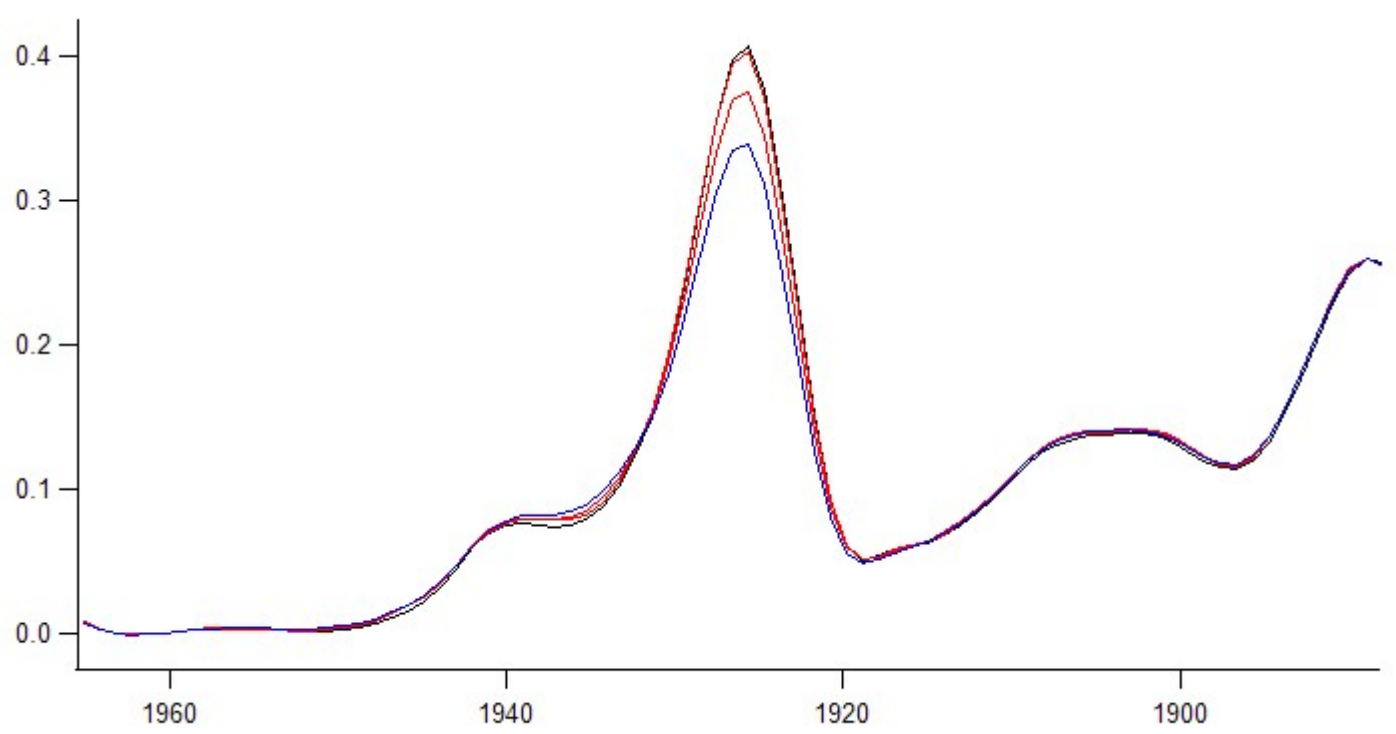

Spectral changes resulting from $355 \mathrm{~nm}$ irradiation of (PNP)Rh(CO) in methylcyclohexane matrix @ 77K. Note: Solvent bands have not been subtracted. Band maximum for (PNP)Rh(CO) is $1925.6 \mathrm{~cm}^{-1}$.

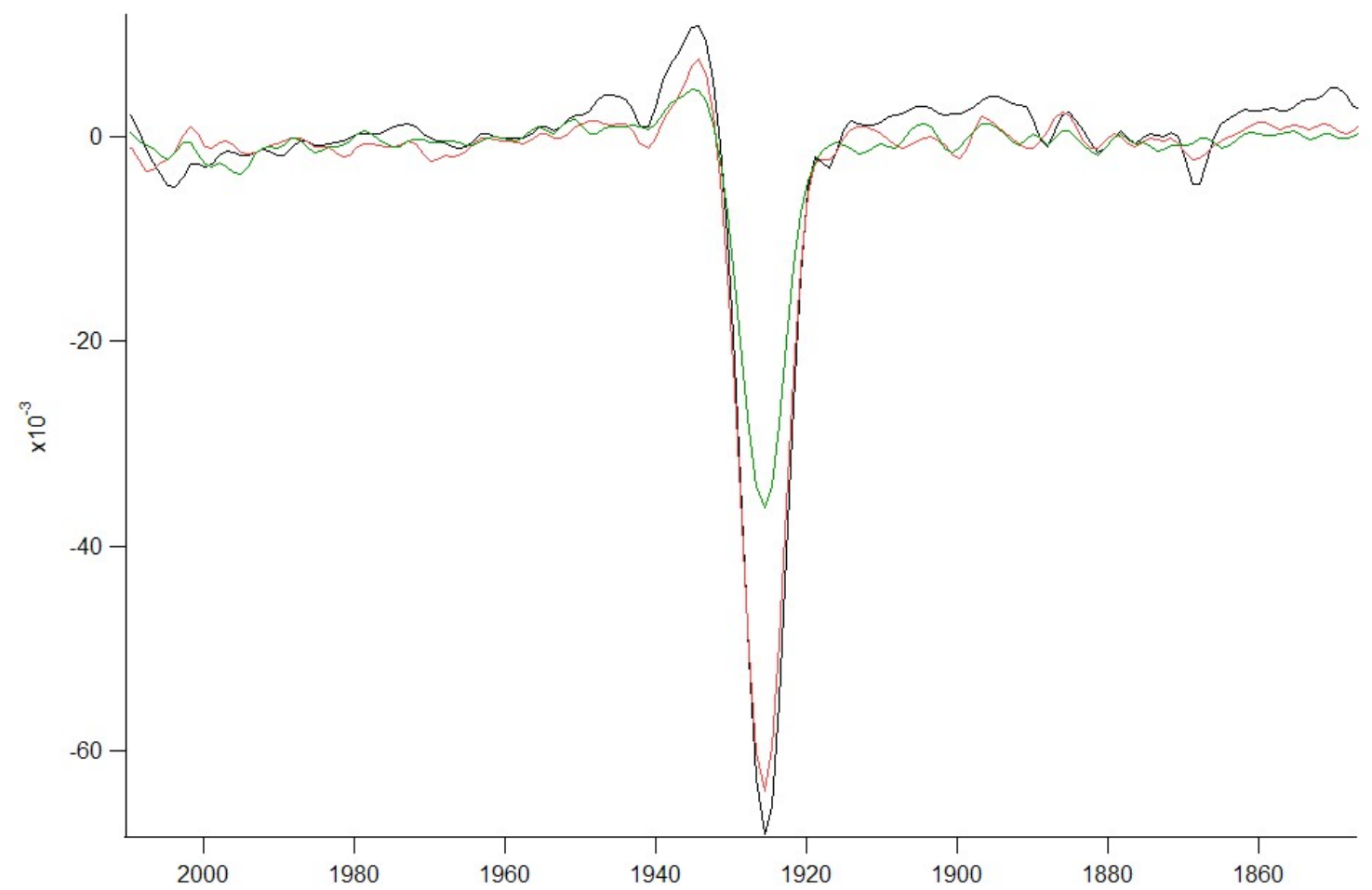

(PNP)Rh(CO) in methylcyclohexane matrix @ 77K. Difference spectra show bleach of band at 1925.6 $\mathrm{cm}^{-1}$.

Time-resolved step-scan FTIR experiments were performed using a Bruker IFS 66/S FTIR spectrometer. The third harmonic of a Nd:YAG laser (355 nm, 6 ns FWHM, $1-2 \mathrm{~mJ} /$ pulse) was used to excite the sample at a repetition rate of $5 \mathrm{~Hz}$. A $1 \mathrm{~mm}$ photovoltaic $\mathrm{HgCdTe}$ infrared detector 
equipped with a $20 \mathrm{MHz}$ pre-amplifier (Kolmar, KMPV11-1-LJ2/239) was used in these experiments. The detector has both AC- and DC-coupled outputs, which were amplified and/or attenuated by an external pre-amplifier and attenuator (Phillips Scientific, model nos. 6931 and 5010), and digitized by an external $100 \mathrm{MHz}$ 8-bit digitizer (Spectrum, PAD82a). Before commencing the laser excitation measurement, the DC output was collected in step-scan mode with Mertz phase correction, in order to generate a set of single beam background spectra and also to store a set of phase spectra in memory, which were later used to automatically phase correct the subsequently recorded time-resolved AC spectra. During the laser flash photolysis, the AC output was monitored and amplified by x60, in order to use the full dynamic range of the digitizer. An optical bandpass filter (OCLI, W04944-8; ca. 2250 $1820 \mathrm{~cm}^{-1}$ bandpass) was used in order to minimize the number of interferogram mirror positions. Single-sided interferograms, with 16 laser shots at each mirror position, were collected at a spectral resolution of $4 \mathrm{~cm}^{-1}$, and a phase resolution of $32 \mathrm{~cm}^{-1}$. Delta absorbance TRIR spectra, $\Delta(\mathrm{OD})_{t}$, at each of the time delays, $t$ following the laser flash, were generated using the equation, $\Delta(O D)_{t}=-\log _{10}\left(1+\gamma(A C)_{t} / D C\right)$, where $\gamma$ is the ratio of the amplifications applied to the DC and AC signals, $(\mathrm{AC})_{t}$ is the single beam spectrum generated from the detector's AC output at time $t$ after the laser flash, and DC is the single beam spectrum that was generated from the DC output before the laser measurement. The solutions were prepared in ca. $20-30 \mathrm{ml}$ of dry n-heptane vacuum distilled into a reservoir vessel using a high-vacuum $\left(10^{-7}\right.$ torr) line. After measuring the $n$-heptane vapor pressure, the solution was then saturated with a known pressure of $\mathrm{CO}$ gas. The concentration of $\mathrm{CO}$ in n-heptane was calculated to be $11.79 \mathrm{mM}$ / atm from previously published data [Wilhelm, E.; Battino, R. Chem. Rev. 1973, 73, 1]. The solutions were handled in an air-sensitive manner and flowed cyclically through an air-tight, home-built flow system consisting of $1 / 16$ " o.d. stainless-steel tubing and Swagelok ${ }^{\circledR}$ fittings, a glass sample reservoir vessel fitted with glass-to-metal $1 / 4$ in. Kovar joints, a $1 \mathrm{~mm}$ pathlength $\mathrm{CaF}_{2}$ IR flow cell (Harrick Scientific Products, DLC-S25) and a recirculating gear pump (Micropump, GA-V23JFSG with HG-0024 drive). 


\section{1 and $2001 \mathrm{~cm}^{-1}$ ) as a function of [CO] following $355 \mathrm{~nm}$ laser flash photolysis of (PNP)Rh(CO)}

\section{in n-heptane}
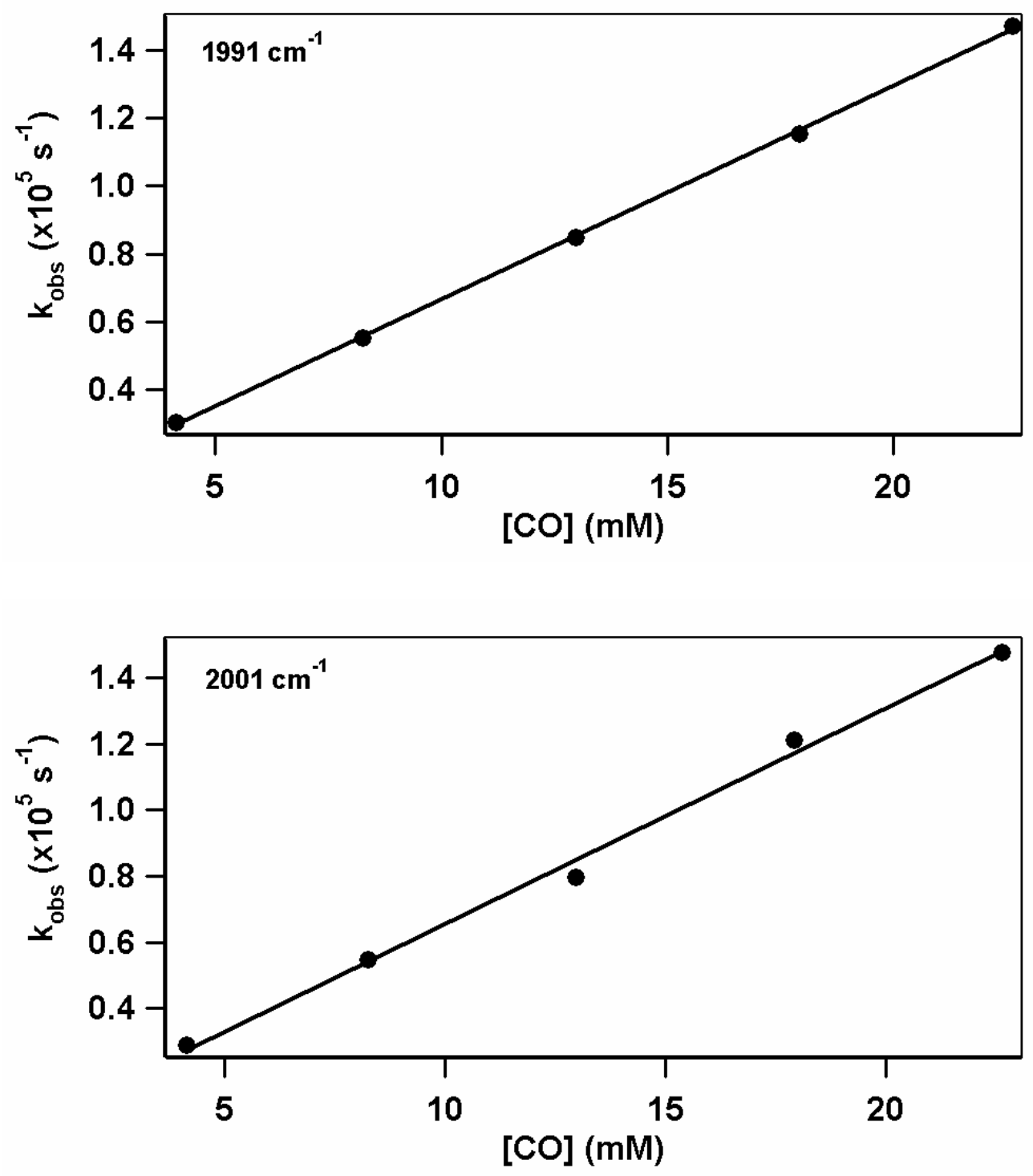

\section{Computational Details}

All calculations were carried out using Density Functional Theory as implemented in the Jaguar 6.0 suite $^{1}$ of $a b$ initio quantum chemistry programs. Geometry optimizations were performed with the PBE $^{2}$ functional and the $6-31 \mathrm{G}^{* *}$ basis set with no symmetry restrictions. Rhodium was represented using the Los Alamos LACVP basis ${ }^{3,4}$. The energies of the optimized structures were reevaluated by additional single-point calculations on each optimized geometry using Dunning's correlation-consistent triple- $\zeta$ basis set ${ }^{5}$ cc-pVTZ(-f) that includes a double set of polarization functions. For all transition metals, we used a modified version of LACVP, designated as LACV3P, in which the exponents were decontracted to match the effective core potential with the triple- $\zeta$ quality basis. 
Vibrational frequency calculations based on analytical second derivatives at the PBE/6-31G** (LACVP) level of theory were carried out on smaller models to compute the stretch frequencies. Zeropoint-energy (ZPE) and entropy corrections at room temperature were derived utilizing unscaled frequencies. The molecules used for vibrational frequency calculations are generated from the corresponding fully optimized structure by replacing all $\mathrm{CH}_{3}$ groups to $\mathrm{H}$ atoms. IR intensities were computed by numerical second derivatives at the same level, and were given in $\mathrm{km} / \mathrm{mol}$.

The models used in this study consist of $\sim 80$ atoms, which represent the non-truncated substrates that were also used in the experimental work.

\section{References}

1. Jaguar, version 6.0, Schrödinger, L.L.C, New York, NY, 2005.

2. Perdew, J. P.; Burke, K.; Ernzerhof, M. Phys. Rev. Lett. 1996, 77, 3865; Phys. Rev. Lett (Erratum) 1997, $78,1386$.

3. Hay, P. J.; Wadt, W. R., J. Chem. Phys. 1985, 82, 270.

4. Wadt, W. R.; Hay, P. J., J. Chem. Phys. 1985, 82, 284.

5. Dunning, T. H., J. Chem. Phys. 1989, 90, 1007. 


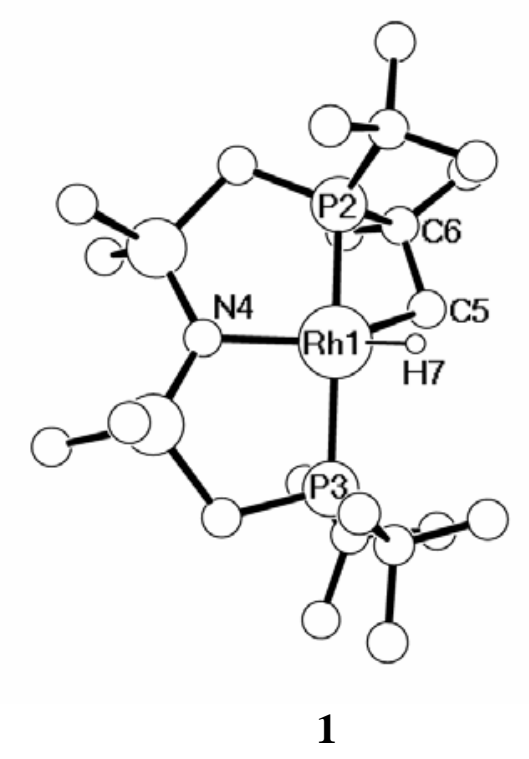

Select bond lengths $(\AA)$ and bond angles $\left(^{\circ}\right)$.

\begin{tabular}{|l|l|}
\hline Rh1-N4 & 2.132 \\
\hline Rh1-P2 & 2.262 \\
\hline Rh1-P3 & 2.379 \\
\hline Rh1-C5 & 2.132 \\
\hline Rh1-H7 & 1.550 \\
\hline P3-Rh1-P2 & 175.3 \\
\hline N4-Rh1-P2 & 90.2 \\
\hline N4-Rh1-P3 & 90.3 \\
\hline N4-Rh1-H7 & 142.9 \\
\hline N4-Rh1-C5 & 140.3 \\
\hline H7-Rh1-C5 & 74.1 \\
\hline Rh1-C5-C6 & 101.5 \\
\hline Rh1-P2-C6 & 86.7 \\
\hline
\end{tabular}

$\mathrm{Rh}-\mathrm{H}$ stretch frequency:

$2123.5 \mathrm{~cm}-1$ (intensity 82.6). 
2. (PNP)Rh (2, non-agostic)

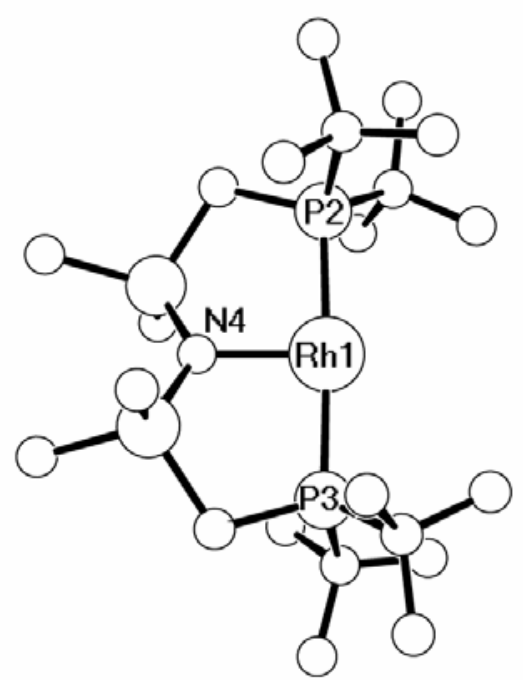

2

$\mathbf{1} \rightarrow \mathbf{2} \Delta \mathrm{E}(\mathrm{SCF})=+2.88 \mathrm{kcal} / \mathrm{mol} \quad \Delta \mathrm{G}^{298.15 \mathrm{~K}}=+3.26 \mathrm{kcal} / \mathrm{mol}$

Select bond lengths $(\AA)$ and bond angles $\left(^{\circ}\right)$.

\begin{tabular}{|l|l|}
\hline Rh1-N4 & 2.048 \\
\hline Rh1-P2 & 2.318 \\
\hline Rh1-P3 & 2.318 \\
\hline P3-Rh1-P2 & 177.6 \\
\hline N4-Rh1-P2 & 88.8 \\
\hline N4-Rh1-P3 & 88.8 \\
\hline
\end{tabular}


3. (PNP)Rh (3, agostic)

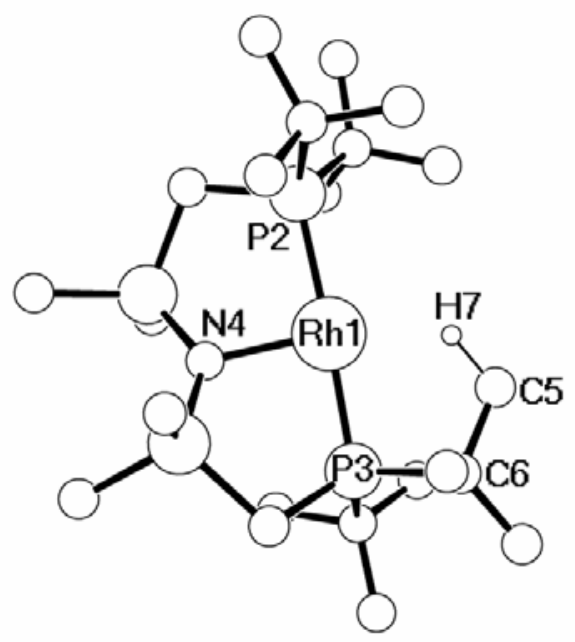

\section{$1 \rightarrow 3 \quad \Delta \mathrm{E}(\mathrm{SCF})=+2.47 \mathrm{kcal} / \mathrm{mol}$}

Select bond lengths $(\AA)$ and bond angles $\left(^{\circ}\right)$.

\begin{tabular}{|l|l|}
\hline Rh1-N4 & 2.061 \\
\hline Rh1-P2 & 2.349 \\
\hline Rh1-P3 & 2.272 \\
\hline Rh1-H7 & 2.10 \\
\hline Rh1-C5 & 2.86 \\
\hline P3-Rh1-P2 & 177.3 \\
\hline N4-Rh1-P2 & 89.9 \\
\hline N4-Rh1-P3 & 87.6 \\
\hline Rh1-P3-C6 & 99.3 \\
\hline
\end{tabular}


4. (PNP)Rh $\left(\mathrm{CH}_{4}\right)$ (4).

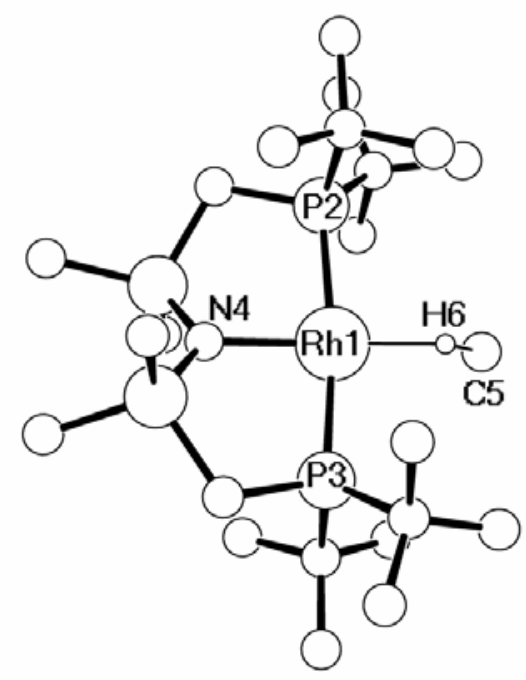

4

$2+\mathrm{CH}_{4} \rightarrow 4 \Delta \mathrm{E}(\mathrm{SCF})=-0.78 \mathrm{kcal} / \mathrm{mol}$

Select bond lengths $(\AA)$ and bond angles $\left(^{\circ}\right)$.

\begin{tabular}{|l|l|}
\hline Rh1-N4 & 2.091 \\
\hline Rh1-P2 & 2.369 \\
\hline Rh1-P3 & 2.392 \\
\hline Rh1-C5 & 2.53 \\
\hline Rh1-H6 & 1.86 \\
\hline C5-H6 & 1.157 \\
\hline P3-Rh1-P2 & 170.8 \\
\hline N4-Rh1-P2 & 85.9 \\
\hline N4-Rh1-P3 & 86.6 \\
\hline N4-Rh1-H6 & 162.1 \\
\hline Rh1-H6-C5 & 112.3 \\
\hline
\end{tabular}




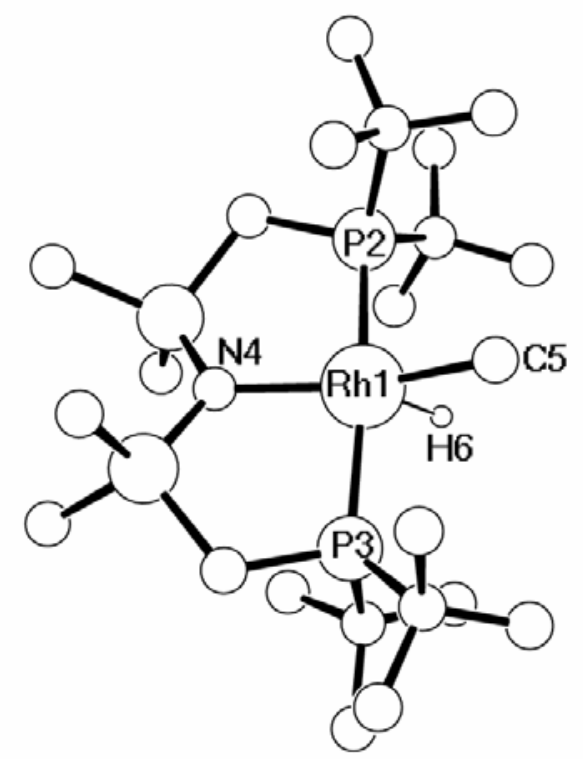

5

$2+\mathrm{CH}_{4} \rightarrow 5 \quad \Delta \mathrm{E}(\mathrm{SCF})=-1.87 \mathrm{kcal} / \mathrm{mol}$

Select bond lengths $(\AA)$ and bond angles $\left({ }^{\circ}\right)$.

\begin{tabular}{|l|l|}
\hline Rh1-N4 & 2.155 \\
\hline Rh1-P2 & 2.375 \\
\hline Rh1-P3 & 2.390 \\
\hline Rh1-C5 & 2.112 \\
\hline Rh1-H6 & 1.531 \\
\hline P3-Rh1-P2 & 171.1 \\
\hline N4-Rh1-P2 & 88.2 \\
\hline N4-Rh1-P3 & 86.8 \\
\hline N4-Rh1-H6 & 131.6 \\
\hline N4-Rh1-C5 & 158.3 \\
\hline H6-Rh1-C5 & 70.1 \\
\hline
\end{tabular}


6. (PNP)Rh(CO) (6).

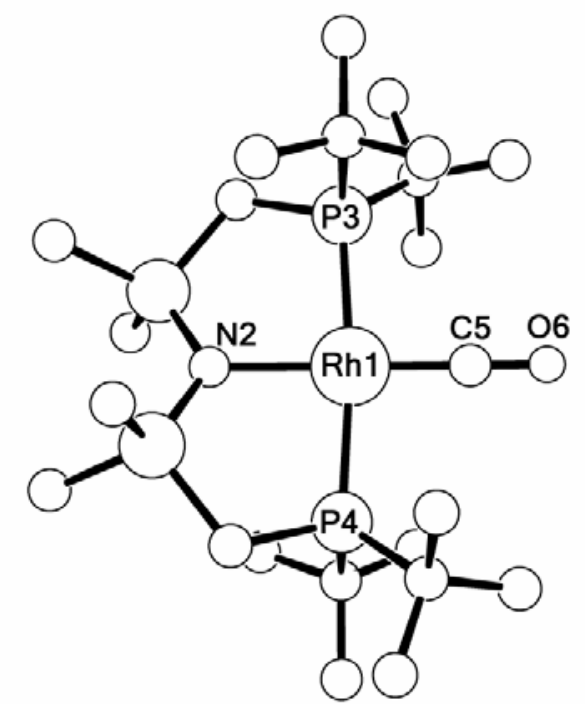

6

$\mathbf{2}+\mathrm{CO} \rightarrow \mathbf{6} \Delta \mathrm{E}(\mathrm{SCF})=-64.04 \mathrm{kcal} / \mathrm{mol}$

$\mathbf{1}+\mathrm{CO} \rightarrow \mathbf{6} \Delta \mathrm{E}(\mathrm{SCF})=-61.16 \mathrm{kcal} / \mathrm{mol}$

CO stretch frequency: $1941.3 \mathrm{~cm}-1$

Select bond lengths $(\AA)$ and bond angles $\left(^{\circ}\right)$.

\begin{tabular}{|l|l|}
\hline Rh1-N2 & 2.129 \\
\hline Rh1-P3 & 2.379 \\
\hline Rh1-P4 & 2.376 \\
\hline Rh1-C5 & 1.825 \\
\hline C5-O6 & 1.184 \\
\hline P3-Rh1-P4 & 173.6 \\
\hline N2-Rh1-C5 & 178.5 \\
\hline Rh1-C5-O6 & 178.8 \\
\hline
\end{tabular}


7. (PNP)Rh(CO) (7 and 8).

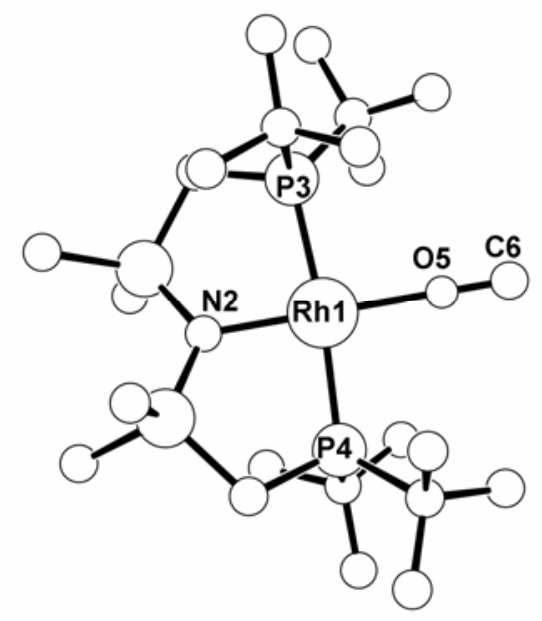

7

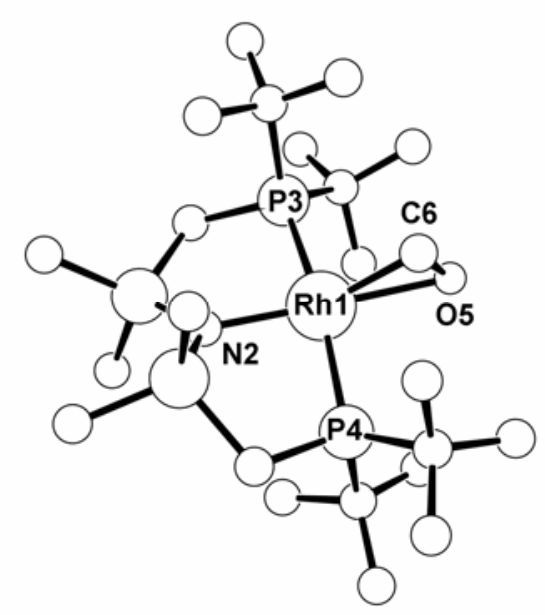

8

$6 \rightarrow 7 \quad \Delta \mathrm{E}(\mathrm{SCF})=+55.87 \mathrm{kcal} / \mathrm{mol}$

$\mathbf{6} \rightarrow \mathbf{8} \quad \Delta \mathrm{E}(\mathrm{SCF})=+41.90 \mathrm{kcal} / \mathrm{mol}$

CO stretch frequency:

7: $1919.8 \mathrm{~cm}-1$

8: $1666.5 \mathrm{~cm}-1$

Select bond lengths $(\AA)$ and bond angles $\left(^{\circ}\right)$.

\begin{tabular}{|l|l|l|l|}
\hline \multicolumn{2}{|c|}{$\mathbf{7}$} & \multicolumn{2}{c|}{$\mathbf{8}$} \\
\hline Rh1-N2 & 2.068 & Rh1-N2 & 2.073 \\
\hline Rh1-P3 & 2.371 & Rh1-P3 & 2.404 \\
\hline Rh1-P4 & 2.369 & Rh1-P4 & 2.375 \\
\hline Rh1-O5 & 2.067 & Rh1-O5 & 2.303 \\
\hline O5-C6 & 1.169 & O5-C6 & 1.213 \\
\hline P3-Rh1-P4 & 174.0 & Rh1-C6 & 2.034 \\
\hline N2-Rh1-O5 & 179.8 & P3-Rh1-P4 & 169.6 \\
\hline Rh1-O5-C6 & 179.4 & N2-Rh1-O5 & 176.4 \\
\hline & & O5-Rh1-C6 & 31.7 \\
\hline & & N2-Rh1-C6 & 144.7 \\
\hline
\end{tabular}


8. (PNP')Rh(H)(CO) (9 and 10).

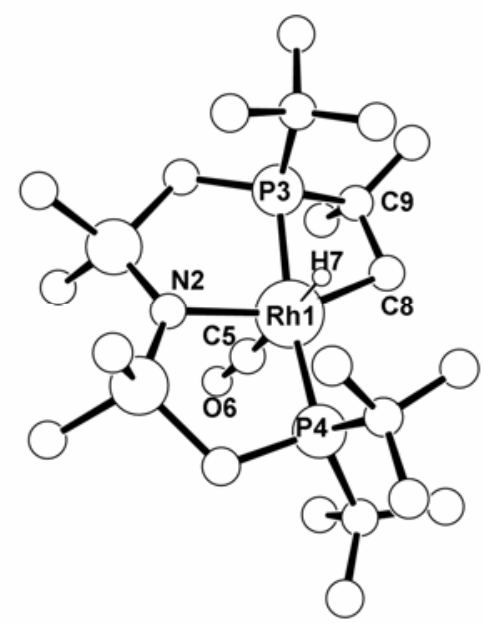

9

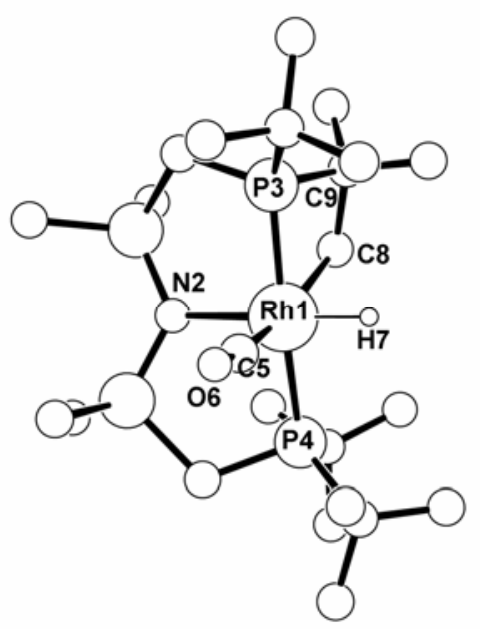

10

$6 \rightarrow 9 \quad \Delta \mathrm{E}(\mathrm{SCF})=+46.63 \mathrm{kcal} / \mathrm{mol}$

$\mathbf{6} \rightarrow \mathbf{1 0} \Delta \mathrm{E}(\mathrm{SCF})=+34.04 \mathrm{kcal} / \mathrm{mol}$

$\begin{array}{llll}\mathbf{1}+\mathbf{C O} \rightarrow \mathbf{9} & \Delta \mathrm{E}(\mathrm{SCF})=\mathbf{- 1 4 . 5 3} \mathrm{kcal} / \mathrm{mol} & \Delta \mathrm{G}^{298.15 \mathrm{~K}}=-2.81 \mathrm{kcal} / \mathrm{mol} \\ \mathbf{1}+\mathbf{C O} \rightarrow \mathbf{1 0} & \Delta \mathrm{E}(\mathrm{SCF})=\mathbf{- 2 7 . 1 2} \mathrm{kcal} / \mathrm{mol} & \Delta \mathrm{G}^{298.15 \mathrm{~K}}=-15.30 \mathrm{kcal} / \mathrm{mol}\end{array}$

Select bond lengths $(\AA)$ and bond angles $\left({ }^{\circ}\right)$.

\begin{tabular}{|l|l|l|l|}
\hline \multicolumn{2}{|c|}{9} & \multicolumn{2}{c|}{$\mathbf{1 0}$} \\
\hline Rh1-N2 & 2.253 & Rh1-N2 & 2.254 \\
\hline Rh1-P3 & 2.271 & Rh1-P3 & 2.324 \\
\hline Rh1-P4 & 2.416 & Rh1-P4 & 2.437 \\
\hline Rh1-C5 & 1.926 & Rh1-C5 & 1.911 \\
\hline C5-O6 & 1.170 & C5-O6 & 1.172 \\
\hline Rh1-C8 & 2.177 & Rh1-C8 & 2.176 \\
\hline Rh1-H7 & 1.610 & Rh1-H7 & 1.592 \\
\hline P3-Rh1-P4 & 159.1 & P3-Rh1-P4 & 167.3 \\
\hline H7-Rh1-C5 & 175.9 & H7-Rh1-N2 & 173.3 \\
\hline Rh1-C5-O6 & 173.4 & Rh1-C5-O6 & 163.5 \\
\hline N2-Rh1-C8 & 156.7 & C5-Rh1-C8 & 162.8 \\
\hline P3-Rh1-C8 & 60.7 & P3-Rh1-C8 & 66.9 \\
\hline Rh1-P3-C9 & 83.6 & Rh1-P3-C9 & 90.2 \\
\hline Rh1-C8-C9 & 102.3 & Rh1-C8-C9 & 105.5 \\
\hline
\end{tabular}

CO stretch frequency:

9: $2024.2 \mathrm{~cm}-1$ (intensity 305.3), strongly coupled with Rh-H at $1916.3 \mathrm{~cm}-1$ (intensity 40.5).

Replace H by D:

$1986.4 \mathrm{~cm}-1$, not coupled with Rh-D at $1390.2 \mathrm{~cm}-1$.

10: $1978.7 \mathrm{~cm}-1$ (intensity 224.5 ), slightly coupled with $\mathrm{Rh}-\mathrm{H}$ at $1973.0 \mathrm{~cm}-1$ (intensity 384.8 ).

Replace H by D:

$1978.2 \mathrm{~cm}-1$, not coupled with Rh-D at $1408.2 \mathrm{~cm}-1$. 
S22 


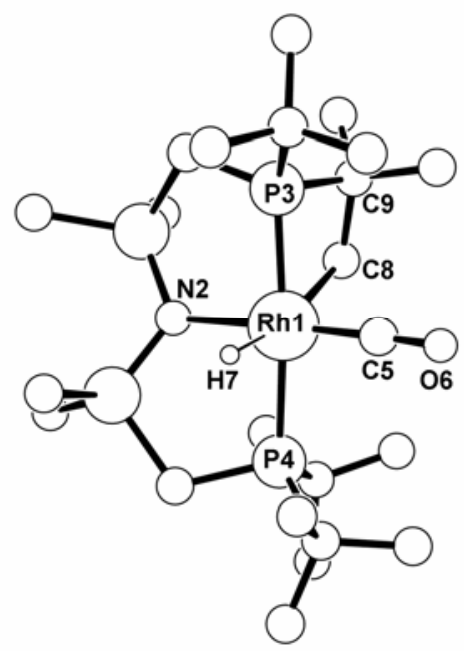

11

$\mathbf{6} \rightarrow \mathbf{1 1} \Delta \mathrm{E}(\mathrm{SCF})=+38.44 \mathrm{kcal} / \mathrm{mol}$

$\mathbf{1}+\mathbf{C O} \rightarrow \mathbf{1 1} \Delta \mathrm{E}(\mathrm{SCF})=\mathbf{- 2 2 . 7 1} \mathrm{kcal} / \mathrm{mol} \quad \Delta \mathrm{G}^{298.15 \mathrm{~K}}=-8.76 \mathrm{kcal} / \mathrm{mol}$

Select bond lengths $(\AA)$ and bond angles $\left(^{\circ}\right)$.

\begin{tabular}{|l|l|}
\hline \multicolumn{2}{|c|}{$\mathbf{1 1}$} \\
\hline Rh1-N2 & 2.191 \\
\hline Rh1-P3 & 2.351 \\
\hline Rh1-P4 & 2.420 \\
\hline Rh1-C5 & 1.841 \\
\hline C5-O6 & 1.176 \\
\hline Rh1-C8 & 2.273 \\
\hline Rh1-H7 & 1.638 \\
\hline P3-Rh1-P4 & 172.3 \\
\hline H7-Rh1-C8 & 166.2 \\
\hline Rh1-C5-O6 & 177.8 \\
\hline N2-Rh1-C5 & 177.3 \\
\hline P3-Rh1-C8 & 66.3 \\
\hline Rh1-P3-C9 & 91.1 \\
\hline Rh1-C8-C9 & 103.7 \\
\hline
\end{tabular}

CO stretch frequency:

11: 1979.9 cm-1 (intensity 455.4), not coupled with Rh-H at $1801.9 \mathrm{~cm}-1$ (intensity 291.0). Replace H by D:

$1979.9 \mathrm{~cm}-1$, not coupled with Rh-D at $1285.8 \mathrm{~cm}-1$. 
Optimized Structures.

\begin{tabular}{|c|c|c|c|}
\hline \\
\hline $\mathrm{Rh}$ & 15.274828945 & 2.464682289 & \\
\hline $\mathrm{P}$ & 16.376046800 & 0.405825818 & 19.149479913 \\
\hline $\mathrm{P}$ & & & \\
\hline $\mathrm{Si}$ & 15.132030744 & & \\
\hline $\mathrm{Si}$ & 12.955699970 & 3.421402349 & 20.885049017 \\
\hline $\mathrm{N}$ & 14.344426603 & 2.404677477 & 20.612479464 \\
\hline $\mathrm{C}$ & & & \\
\hline $\mathrm{H}$ & & & 23.45467042 \\
\hline $\mathrm{H}$ & 17.079732638 & 3.000773438 & 22.220426085 \\
\hline $\mathrm{H}$ & 16.93 & 1.8447 & \\
\hline $\mathrm{C}$ & & & \\
\hline $\mathrm{H}$ & & & \\
\hline $\mathrm{H}$ & 14.5 & -0.0 & \\
\hline $\mathrm{H}$ & & & \\
\hline $\mathrm{C}$ & & & \\
\hline $\mathrm{H}$ & 16.990597778 & & 21.4688145 \\
\hline $\mathrm{H}$ & & & \\
\hline $\mathrm{C}$ & & & \\
\hline $\mathrm{C}$ & & & \\
\hline $\mathrm{C}$ & & & \\
\hline $\mathrm{H}$ & & & \\
\hline $\mathrm{H}$ & & & \\
\hline $\mathrm{H}$ & 12.1 & 5.17 & 6023 \\
\hline $\mathrm{C}$ & & & \\
\hline $\mathrm{H}$ & & & \\
\hline $\mathrm{H}$ & & & \\
\hline $\mathrm{H}$ & & & 92941 \\
\hline $\mathrm{C}$ & & & \\
\hline $\mathrm{H}$ & & & \\
\hline $\mathrm{H}$ & 12.6 & & \\
\hline $\mathrm{C}$ & & & \\
\hline $\mathrm{C}$ & & & \\
\hline $\mathrm{H}$ & & & \\
\hline $\mathrm{H}$ & & & \\
\hline & & & \\
\hline $\mathrm{C}$ & & & \\
\hline $\mathrm{C}$ & & -2.505 & 18.903955810 \\
\hline $\mathrm{C}$ & & & \\
\hline & & & \\
\hline $\mathrm{C}$ & & & \\
\hline $\mathrm{C}$ & & & 20.0 \\
\hline $\mathrm{C}$ & & & \\
\hline & & & \\
\hline $\mathrm{C}$ & 12.252121506 & & \\
\hline $\mathrm{C}$ & 14.769480845 & 2.4282056 & 16.624274248 \\
\hline & & & \\
\hline & & & \\
\hline $\mathrm{H}$ & 19.7823913 & 1.837507 & 19.890194372 \\
\hline & & & \\
\hline $\mathrm{H}$ & & & \\
\hline & & & \\
\hline $\mathrm{H}$ & 18.903347552 & -1.563129648 & 18.612043032 \\
\hline & $18 . / 01 / 80852$ & -1.202157950 & 20.55054081 \\
\hline
\end{tabular}




\begin{tabular}{crrr}
$\mathrm{H}$ & 19.784660037 & 1.181968697 & 17.588436229 \\
$\mathrm{H}$ & 18.161151396 & 1.877993112 & 17.306449320 \\
$\mathrm{H}$ & 18.510774484 & 0.192100274 & 16.839793293 \\
$\mathrm{H}$ & 15.597804327 & -3.330193569 & 18.382953563 \\
$\mathrm{H}$ & 15.835291353 & -2.564367308 & 19.967295686 \\
$\mathrm{H}$ & 17.198046873 & -2.698024032 & 18.829630122 \\
$\mathrm{H}$ & 15.547361129 & -1.922923092 & 16.222743764 \\
$\mathrm{H}$ & 17.199602127 & -1.441097152 & 16.659785256 \\
$\mathrm{H}$ & 15.976342333 & -0.192531613 & 16.295532976 \\
$\mathrm{H}$ & 13.700829978 & -1.893755264 & 17.813948169 \\
$\mathrm{H}$ & 13.817689713 & -0.105520454 & 17.858516042 \\
$\mathrm{H}$ & 13.800661746 & -1.034441228 & 19.372044240 \\
$\mathrm{H}$ & 16.003451100 & 7.412096541 & 19.194062825 \\
$\mathrm{H}$ & 16.112254470 & 5.709535571 & 19.736119947 \\
$\mathrm{H}$ & 14.619478071 & 6.648887714 & 20.011509174 \\
$\mathrm{H}$ & 16.680149509 & 6.837073446 & 16.910479131 \\
$\mathrm{H}$ & 15.866551818 & 5.576860485 & 15.953665357 \\
$\mathrm{H}$ & 16.849936835 & 5.114625221 & 17.366717397 \\
$\mathrm{H}$ & 13.196828441 & 3.472873909 & 14.448505396 \\
$\mathrm{H}$ & 14.31189640 & 4.743880297 & 14.990541030 \\
$\mathrm{H}$ & 12.558862445 & 4.895806175 & 15.309525615 \\
$\mathrm{H}$ & 15.567928170 & 2.756225072 & 15.939741160 \\
$\mathrm{H}$ & 12.060533654 & 1.939244079 & 16.053264645 \\
$\mathrm{H}$ & 11.380580952 & 3.326334224 & 16.938562135 \\
$\mathrm{H}$ & 12.316813976 & 2.073771994 & 17.813272930 \\
$\mathrm{H}$ & 14.468958387 & 1.401368899 & 16.355309033 \\
$\mathrm{H}$ & 16.527995989 & 2.981399561 & 17.944025951 \\
\hline & & &
\end{tabular}

2

\begin{tabular}{|c|c|c|c|}
\hline $\mathrm{Rh}$ & 15.427940848 & 2.183161376 & 18.6895 \\
\hline $\mathrm{P}$ & & & \\
\hline $\mathrm{P}$ & 14.302274148 & 4.103062179 & 18.041201977 \\
\hline $\mathrm{Si}$ & & & \\
\hline $\mathrm{Si}$ & & & \\
\hline $\mathrm{N}$ & 14.509841398 & & \\
\hline $\mathrm{C}$ & 16.755412235 & & \\
\hline $\mathrm{H}$ & & & \\
\hline $\mathrm{H}$ & & & \\
\hline $\mathrm{H}$ & & & \\
\hline $\mathrm{C}$ & & & \\
\hline $\mathrm{H}$ & & & \\
\hline $\mathrm{H}$ & & & \\
\hline $\mathrm{H}$ & & & \\
\hline $\mathrm{C}$ & & & \\
\hline $\mathrm{H}$ & & & \\
\hline $\mathrm{H}$ & & & \\
\hline $\mathrm{C}$ & & & \\
\hline $\mathrm{C}$ & & & \\
\hline $\mathrm{C}$ & & & \\
\hline $\mathrm{H}$ & & & \\
\hline & & & \\
\hline $\mathrm{H}$ & & & \\
\hline $\mathrm{C}$ & & & \\
\hline $\mathrm{H}$ & & & 18.975962269 \\
\hline & & & \\
\hline $\mathrm{H}$ & & & \\
\hline $\mathrm{C}$ & 12.868850751 & 4.383805812 & 19.193971722 \\
\hline $\mathrm{H}$ & 11.917633875 & 4.458966114 & 18.641080711 \\
\hline
\end{tabular}




\begin{tabular}{|c|c|c|c|}
\hline & 13.002528362 & 5.335797358 & \\
\hline & 15.298245010 & 5.749470941 & 18.146320493 \\
\hline & 14.457903524 & 7.029586628 & \\
\hline & 15.090849937 & 7.910196053 & \\
\hline & 13.608403965 & 7.062501602 & 18.685694860 \\
\hline & 14.060314559 & & \\
\hline & 13.558253162 & 3.845605259 & \\
\hline & 16.284536725 & -2.648484875 & 19.607375003 \\
\hline & 15.887187076 & -1.576550601 & 17.355443305 \\
\hline & & & \\
\hline & & -0.913195030 & \\
\hline $\mathrm{C}$ & 18.968025885 & & \\
\hline $\mathrm{C}$ & 18.430884410 & 0.787334587 & \\
\hline & & & \\
\hline & 12.465744166 & & \\
\hline & 14.678453378 & 3.246836745 & 53837 \\
\hline & 15. & & \\
\hline & & & \\
\hline & 19.9 & & \\
\hline & 19.0 & & \\
\hline & 18. & & \\
\hline & & & \\
\hline $\mathrm{H}$ & & -1.72 & 18.507295103 \\
\hline & & & \\
\hline & 19. & & 5880 \\
\hline & 17.876647602 & & 17.314799064 \\
\hline $\mathrm{H}$ & 18.014857741 & 0.029959583 & 16.784499761 \\
\hline & & & \\
\hline & & & \\
\hline $\mathrm{H}$ & & -2.86 & 37717 \\
\hline $\mathrm{H}$ & & & \\
\hline & & & \\
\hline & & & \\
\hline $\mathrm{H}$ & & -2.1 & 88381 \\
\hline & & & \\
\hline & & & 7067 \\
\hline $\mathrm{H}$ & 16.576703487 & 6.613987654 & 19.683203471 \\
\hline $\mathrm{H}$ & 16.518866787 & & 1063485 \\
\hline & & & \\
\hline П & & & \\
\hline $\mathrm{H}$ & 16.073 & 5.84 & 16.07 \\
\hline $\mathrm{H}$ & & & \\
\hline & & & \\
\hline $\mathrm{H}$ & & & \\
\hline $\mathrm{H}$ & 12.209586204 & 5.584608423 & 16.275386100 \\
\hline & & & 14.415996884 \\
\hline & & & \\
\hline & & & 15.245960249 \\
\hline $\mathrm{H}$ & 12.121659727 & 2.455258082 & 15.432892526 \\
\hline & & & \\
\hline & 12.867515201 & 1.877536043 & 16.95693632 \\
\hline
\end{tabular}

$\begin{array}{llll}======================== \\ 3 & & \\ ========================= \\ \text { Rh } & 15.286472562 & 2.209082628 & 18.591538832 \\ \text { P } & 16.410598397 & 0.249460582 & 19.233735623 \\ \text { P } & 14.204396796 & 4.138374421 & 18.073341078 \\ \text { Si } & 15.658142559 & 1.848172536 & 21.751443370 \\ \text { Si } & 12.932465687 & 2.991970725 & 20.544132224\end{array}$


$\begin{array}{llll}\mathrm{N} & 14.583731883 & 2.433666929 & 20.515748454\end{array}$

C $\quad 17.041210739 \quad 3.070990255 \quad 22.217429759$

$\begin{array}{lllll}\mathrm{H} & 16.604641827 & 3.959891907 & 22.705065471\end{array}$

$\begin{array}{lllll}\mathrm{H} & 17.599554682 & 3.417653986 & 21.334041592\end{array}$

$\begin{array}{lllll}\mathrm{H} & 17.761650868 & 2.623608745 & 22.925574867\end{array}$

C $\quad 14.771491404 \quad 1.425161243 \quad 23.389443363$

$\begin{array}{lllll}\mathrm{H} & 14.400314473 & 2.328081821 & 23.901356104\end{array}$

$\begin{array}{lllll}\mathrm{H} & 15.472259311 & 0.922975727 & 24.079630395\end{array}$

$\begin{array}{lllll}\mathrm{H} & 13.914735943 & 0.748313353 & 23.231694911\end{array}$

$\begin{array}{lllll}\text { C } & 16.415141376 & 0.205051044 & 21.098413187\end{array}$

H $\quad 17.418037108-0.018009909 \quad 21.501324951$

$\begin{array}{llll}\mathrm{H} & 15.749530803 & -0.608651139 & 21.435986192\end{array}$

$\begin{array}{llll}\text { C } & 15.592552878 & -1.430957061 & 18.752793695\end{array}$

$\begin{array}{llll}\text { C } & 18.266518064 & 0.283219758 & 18.725210172\end{array}$

$\begin{array}{lllll}\text { C } & 12.464074116 & 3.864755790 & 22.175899866\end{array}$

$\begin{array}{llll}\mathrm{H} & 13.178121322 & 4.666475038 & 22.429104200\end{array}$

$\begin{array}{lllll}\mathrm{H} & 12.429533551 & 3.163037970 & 23.024907377\end{array}$

$\begin{array}{lllll}\mathrm{H} & 11.462587638 & 4.320869160 & 22.085943146\end{array}$

$\begin{array}{llll}\text { C } & 11.637663641 & 1.624991072 & 20.257430465\end{array}$

$\begin{array}{lllll}\mathrm{H} & 11.786337603 & 1.143767333 & 19.276385650\end{array}$

$\begin{array}{llll}\mathrm{H} & 10.607949806 & 2.023283009 & 20.294931236\end{array}$

$\begin{array}{lllll}\mathrm{H} & 11.722845700 & 0.837905235 & 21.026410811\end{array}$

$\begin{array}{llll}\text { C } & 12.680587054 & 4.282932148 & 19.116387784\end{array}$

$\begin{array}{lllll}\mathrm{H} & 11.794356888 & 3.996580126 & 18.527356954\end{array}$

$\begin{array}{lllll}\mathrm{H} & 12.513665471 & 5.308471647 & 19.488656341\end{array}$

$\begin{array}{lllll}\text { C } & 15.122452264 & 5.800117797 & 18.310244442\end{array}$

C $\quad 14.303286191 \quad 7.032181901 \quad 17.886348986$

$\begin{array}{lllll}\mathrm{H} & 14.845546467 & 7.950762908 & 18.177265598\end{array}$

$\begin{array}{lllll}\mathrm{H} & 13.320135837 & 7.063385409 & 18.385769794\end{array}$

$\begin{array}{lllll}\mathrm{H} & 14.136164760 & 7.076472427 & 16.799993649\end{array}$

$\begin{array}{llll}\text { C } & 13.811520044 & 3.786224387 & 16.217671222\end{array}$

$\begin{array}{llll}\text { C } & 16.101764731 & -2.654396740 & 19.537765142\end{array}$

$\begin{array}{llll}\text { C } & 15.742652980 & -1.681675918 & 17.240535038\end{array}$

$\begin{array}{llll}\text { C } & 14.093166269 & -1.221410780 & 19.063587974\end{array}$

$\begin{array}{llll}\text { C } & 19.059873228 & -0.992127156 & 19.054814606\end{array}$

$\begin{array}{llll}\text { C } & 18.885071132 & 1.481947134 & 19.476348143\end{array}$

$\begin{array}{llll}\text { C } & 18.354399262 & 0.596829255 & 17.216509606\end{array}$

$\begin{array}{llll}\text { C } & 13.665837997 & 4.968265972 & 15.250825892\end{array}$

$\begin{array}{llll}\text { C } & 12.534122434 & 2.926778286 & 16.162307538\end{array}$

$\begin{array}{llll}\text { C } & 15.022315512 & 2.894178529 & 15.830501398\end{array}$

$\begin{array}{llll}\text { C } & 15.431074260 & 5.884225420 & 19.822172978\end{array}$

C $\quad 16.459001385 \quad 5.735619194 \quad 17.545430125$

$\begin{array}{llll}\mathrm{H} & 19.915492197 & 1.648644224 & 19.111636770\end{array}$

$\begin{array}{lllll}\mathrm{H} & 18.939234082 & 1.319744720 & 20.564552528\end{array}$

$\mathrm{H} \quad 18.295667432 \quad 2.397628935 \quad 19.291712382$

H $\quad 20.137387970-0.816095680 \quad 18.876067023$

H $\quad 18.760239687-1.842879674 \quad 18.422115205$

H $\quad 18.946072327 \quad-1.290272763 \quad 20.110830659$

$\begin{array}{lllll}\mathrm{H} & 19.412019634 & 0.751920028 & 16.933129343\end{array}$

$\begin{array}{lllll}\mathrm{H} & 17.801186274 & 1.523997911 & 16.983180111\end{array}$

H $\quad 17.954698839 \quad-0.210830399 \quad 16.585484505$

$\begin{array}{lllll}\mathrm{H} & 15.481427387 & -3.533680843 & 19.282994869\end{array}$

H $\quad 16.027699921 \quad-2.510598725 \quad 20.628092485$

H $\quad 17.145421291-2.907723672 \quad 19.300643735$

H $\quad 15.082191549-2.514766952 \quad 16.936396302$

H $\quad 16.771595971 \quad-1.964157426 \quad 16.963924679$

H $\quad 15.452396632 \quad-0.793389435 \quad 16.653113967$

$\mathrm{H} \quad 13.525649565 \quad-2.125461890 \quad 18.773687330$

H $\quad 13.698023138-0.350915414 \quad 18.513035536$ 


$\begin{array}{rrrr}\mathrm{H} & 13.909133666 & -1.039042223 & 20.135790920 \\ \mathrm{H} & 16.093900262 & 6.749309013 & 20.008900010 \\ \mathrm{H} & 15.927978062 & 4.968122941 & 20.179155847 \\ \mathrm{H} & 14.518777551 & 6.030244955 & 20.423922801 \\ \mathrm{H} & 17.070913590 & 6.623578151 & 17.788256488 \\ \mathrm{H} & 16.320723793 & 5.721306372 & 16.451165876 \\ \mathrm{H} & 17.024104062 & 4.833118625 & 17.837772391 \\ \mathrm{H} & 13.457058934 & 4.589692707 & 14.232066183 \\ \mathrm{H} & 14.577880784 & 5.583008385 & 15.190095997 \\ \mathrm{H} & 12.825773313 & 5.622505972 & 15.536722517 \\ \mathrm{H} & 14.895040672 & 2.469121649 & 14.814919873 \\ \mathrm{H} & 15.063980822 & 1.988921326 & 16.512945884 \\ \mathrm{H} & 15.980454055 & 3.434985662 & 15.855717717 \\ \mathrm{H} & 12.425913595 & 2.493050772 & 15.150779635 \\ \mathrm{H} & 11.628122388 & 3.521849003 & 16.364227165 \\ \mathrm{H} & 12.583114900 & 2.096598392 & 16.888203815\end{array}$

4

$\begin{array}{crrr}========================= \\ \mathrm{Rh} & 15.561863425 & 2.237229807 & 18.607454084 \\ \mathrm{P} & 16.614585806 & 0.287876294 & 19.509357802 \\ \mathrm{P} & 14.213080849 & 4.055227303 & 17.910664974 \\ \mathrm{Si} & 15.520986235 & 1.947459562 & 21.807706804 \\ \mathrm{Si} & 12.899187551 & 2.906427457 & 20.431280244 \\ \mathrm{~N} & 14.554657785 & 2.384370987 & 20.433728090 \\ \mathrm{C} & 16.871100545 & 3.221070792 & 22.238839583 \\ \mathrm{H} & 16.410787476 & 4.169065826 & 22.567877770 \\ \mathrm{H} & 17.496691570 & 3.447225764 & 21.359390071 \\ \mathrm{H} & 17.529338316 & 2.870011960 & 23.053941166 \\ \mathrm{C} & 14.552225094 & 1.629997728 & 23.423354547 \\ \mathrm{H} & 14.177015880 & 2.558630857 & 23.881913136 \\ \mathrm{H} & 15.225119209 & 1.151257400 & 24.157205522 \\ \mathrm{H} & 13.694134437 & 0.953744799 & 23.270209454 \\ \mathrm{C} & 16.371944067 & 0.298200148 & 21.355528387 \\ \mathrm{H} & 17.337944879 & 0.193026845 & 21.873186745 \\ \mathrm{H} & 15.743930790 & -0.551604982 & 21.671456503 \\ \mathrm{C} & 15.673062278 & -1.284843591 & 18.901326877 \\ \mathrm{C} & 18.538956462 & -0.004945661 & 19.387780380 \\ \mathrm{C} & 12.314464001 & 3.774019342 & 22.030539173 \\ \mathrm{H} & 13.022539139 & 4.552866125 & 22.361166981 \\ \mathrm{H} & 12.165253797 & 3.073257968 & 22.866940156 \\ \mathrm{H} & 11.343679298 & 4.265422157 & 21.838968411 \\ \mathrm{C} & 11.625266096 & 1.528343318 & 20.086948979 \\ \mathrm{H} & 11.780194214 & 1.071052113 & 19.095050510 \\ \mathrm{H} & 10.592893913 & 1.920359524 & 20.123063815 \\ \mathrm{H} & 11.701811892 & 0.721620879 & 20.836231436 \\ \mathrm{C} & 12.726079118 & 4.182711399 & 19.020757491 \\ \mathrm{H} & 11.817164708 & 3.959168517 & 18.440451020 \\ \mathrm{H} & 12.611040856 & 5.208555198 & 19.409932576 \\ \mathrm{C} & 15.112158501 & 5.744149982 & 18.164845021 \\ \mathrm{C} & 14.336484625 & 6.976477380 & 17.670420897 \\ \mathrm{H} & 14.848539361 & 7.894226149 & 18.015821910 \\ \mathrm{H} & 13.306100465 & 7.008374896 & 18.062818151 \\ \mathrm{H} & 14.290813651 & 7.025742592 & 16.570382622 \\ \mathrm{C} & 13.351332295 & 3.981257841 & 16.165241494 \\ \mathrm{C} & 16.243813580 & -2.619544901 & 19.409033803 \\ \mathrm{C} & 15.630032197 & -1.272891233 & 17.359428056 \\ \mathrm{C} & 14.222253196 & -1.124924125 & 19.405729674 \\ \mathrm{C} & 18.971820558 & -0.394907013 & 17.961261418\end{array}$




\begin{tabular}{cccc} 
C & 19.094788433 & -1.075154069 & 20.352686042 \\
C & 19.175779763 & 1.350815817 & 19.767741740 \\
C & 12.201205674 & 4.994434144 & 15.974878445 \\
C & 12.765900429 & 2.552203499 & 16.082499646 \\
C & 14.346545821 & 4.175788520 & 15.006268096 \\
C & 15.349026275 & 5.860958921 & 19.687504465 \\
C & 16.492870105 & 5.676678022 & 17.483125120 \\
H & 20.196670132 & -1.091098676 & 20.259437408 \\
H & 18.731255934 & -2.085970246 & 20.121803383 \\
H & 18.867074594 & -0.865667559 & 21.409179313 \\
H & 20.076416283 & -0.407139560 & 17.908515721 \\
H & 18.614099182 & 0.312755809 & 17.201667572 \\
H & 18.624341282 & -1.403949146 & 17.687177071 \\
H & 20.273933903 & 1.290946853 & 19.651014406 \\
H & 18.967669183 & 1.618462724 & 20.816840998 \\
H & 18.803069260 & 2.176851172 & 19.140416006 \\
H & 15.539034662 & -3.435676506 & 19.162233780 \\
H & 16.380984089 & -2.624046377 & 20.503596346 \\
H & 17.206969741 & -2.870703683 & 18.936229476 \\
H & 15.017411935 & -2.121422045 & 17.002800898 \\
H & 16.626647589 & -1.366603507 & 16.900408914 \\
H & 15.167325880 & -0.337405985 & 17.001402616 \\
H & 13.587272789 & -1.893923267 & 18.928187449 \\
H & 13.828275892 & -0.127245698 & 19.151969898 \\
H & 14.135798642 & -1.257900560 & 20.496468527 \\
H & 16.035800280 & 6.705626021 & 19.881513784 \\
H & 15.798265298 & 4.938093951 & 20.090141541 \\
H & 14.418314868 & 6.057738759 & 20.244364375 \\
H & 17.057757784 & 6.601514442 & 17.702115080 \\
H & 16.423520111 & 5.583227191 & 16.387284896 \\
H & 17.066747866 & 4.819040187 & 17.871743762 \\
H & 11.680386425 & 4.760496751 & 15.027812556 \\
H & 12.559193016 & 6.031234030 & 15.903529227 \\
H & 11.447874589 & 4.950864477 & 16.776326567 \\
H & 13.810037091 & 4.062143131 & 14.045869228 \\
H & 15.152124722 & 3.428507085 & 15.018839352 \\
H & 14.798955248 & 5.180844982 & 15.007050385 \\
H & 12.315033439 & 2.394484154 & 15.085016712 \\
H & 11.975333110 & 2.384040695 & 16.833295035 \\
H & 13.543289871 & 1.786973478 & 16.247778968 \\
H & 15.937417998 & 1.921479154 & 16.818363320 \\
C & 17.009832721 & 2.246179315 & 16.531517244 \\
H & 17.385078851 & 1.388042126 & 15.956847145 \\
H & 17.680899091 & 2.455620329 & 17.375920464 \\
H & 16.942016729 & 3.133690072 & 15.893946067 \\
\hline & 150
\end{tabular}

5

\begin{tabular}{cccc}
\hline $\mathrm{Rh}$ & 15.703539771 & 2.378745548 & 18.670663637 \\
$\mathrm{P}$ & 16.754748935 & 0.379048041 & 19.451327682 \\
$\mathrm{P}$ & 14.342231013 & 4.178253483 & 17.928243936 \\
$\mathrm{Si}$ & 15.170600322 & 1.524696998 & 21.885918944 \\
$\mathrm{Si}$ & 12.948363735 & 3.037769685 & 20.479704549 \\
$\mathrm{~N}$ & 14.535577355 & 2.329453060 & 20.480568027 \\
$\mathrm{C}$ & 16.014362902 & 2.727579109 & 23.101232900 \\
$\mathrm{H}$ & 15.295569490 & 3.464448854 & 23.498153682 \\
$\mathrm{H}$ & 16.819139965 & 3.290107519 & 22.596870896 \\
$\mathrm{H}$ & 16.456075570 & 2.194618491 & 23.962266336 \\
$\mathrm{C}$ & 13.914990376 & 0.514334873 & 22.918685304
\end{tabular}


$\mathrm{H} \quad 13.165763802 \quad 1.163446760 \quad 23.402308819$

$\begin{array}{llll}\mathrm{H} & 14.435562327 & -0.033374120 & 23.724432300\end{array}$

$\begin{array}{lllll}\mathrm{H} & 13.367441102 & -0.224763413 & 22.310170887\end{array}$

$\begin{array}{llll}\text { C } & 16.532744589 & 0.313795610 & 21.294263851\end{array}$

$\begin{array}{lllll}\mathrm{H} & 17.482441983 & 0.650422108 & 21.744889183\end{array}$

$\begin{array}{lllll}\mathrm{H} & 16.367493722 & -0.719346699 & 21.647830758\end{array}$

$\begin{array}{lllll}\text { C } & 15.799182304 & -1.174642170 & 18.819534664\end{array}$

$\begin{array}{llll}\text { C } & 18.666727895 & 0.079745244 & 19.294399221\end{array}$

$\begin{array}{llll}\text { C } & 12.461113918 & 3.930008616 & 22.101382931\end{array}$

$\begin{array}{lllll}\mathrm{H} & 13.216840928 & 4.681765038 & 22.387389758\end{array}$

$\begin{array}{llll}\mathrm{H} & 12.344733628 & 3.236511432 & 22.950878296\end{array}$

$\begin{array}{lllll}\mathrm{H} & 11.497303758 & 4.455180871 & 21.976850751\end{array}$

C $\quad 11.548411474 \quad 1.796558996 \quad 20.115557595$

$\begin{array}{lllll}\mathrm{H} & 11.728196168 & 1.239847586 & 19.181557096\end{array}$

$\begin{array}{lllll}\mathrm{H} & 10.570456266 & 2.303121593 & 20.029032487\end{array}$

$\begin{array}{lllll}\mathrm{H} & 11.466346677 & 1.057604715 & 20.930862682\end{array}$

$\begin{array}{llll}\text { C } & 12.966195249 & 4.417634345 & 19.157695653\end{array}$

$\begin{array}{lllll}\mathrm{H} & 11.994724235 & 4.566145348 & 18.656018657\end{array}$

$\begin{array}{llll}\mathrm{H} & 13.171272617 & 5.346317892 & 19.715540406\end{array}$

$\begin{array}{llll}\text { C } & 15.142525097 & 5.950039890 & 17.896928788\end{array}$

$\begin{array}{llll}\text { C } & 14.122262639 & 7.106825035 & 17.965844909\end{array}$

$\begin{array}{lllll}\mathrm{H} & 14.681043044 & 8.060014708 & 18.004257457\end{array}$

$\begin{array}{llll}\mathrm{H} & 13.487347174 & 7.072051822 & 18.864319386\end{array}$

$\begin{array}{lllll}\mathrm{H} & 13.463813903 & 7.147664232 & 17.086326928\end{array}$

$\begin{array}{lllll}\text { C } & 13.409471220 & 3.819276301 & 16.282395388\end{array}$

$\begin{array}{llll}\text { C } & 16.384355845 & -2.515677571 & 19.302368734\end{array}$

C $\quad 15.734626808 \quad-1.174642720 \quad 17.278578313$

$\begin{array}{llll}\text { C } & 14.359465431 & -1.039517350 & 19.362388650\end{array}$

$\begin{array}{llll}\text { C } & 19.064813735 & -0.326069839 & 17.861111207\end{array}$

$\begin{array}{llll}\text { C } & 19.212212847 & -0.988156994 & 20.268587032\end{array}$

$\begin{array}{llll}\text { C } & 19.334784025 & 1.426216406 & 19.658960629\end{array}$

$\begin{array}{lllll}\text { C } & 12.520175159 & 4.984996764 & 15.808258721\end{array}$

$\begin{array}{llll}\text { C } & 12.522739772 & 2.585375085 & 16.562144794\end{array}$

$\begin{array}{llll}\text { C } & 14.401055535 & 3.444144680 & 15.163488299\end{array}$

$\begin{array}{llll}\text { C } & 16.021064284 & 5.996859522 & 19.168539048\end{array}$

$\begin{array}{llll}\text { C } & 16.028131691 & 6.176760442 & 16.656699832\end{array}$

$\begin{array}{llll}\text { H } & 20.311836673 & -1.030009228 & 20.158821303\end{array}$

H $\quad 18.824490270-1.995831135 \quad 20.065258793$

H $\quad 19.001305478-0.747354252 \quad 21.321942628$

H $\quad 20.166195643 \quad-0.301023046 \quad 17.768042025$

$\begin{array}{lllll}\mathrm{H} & 18.651600020 & 0.348383946 & 17.096033608\end{array}$

$\begin{array}{lllll}\mathrm{H} & 18.742736137 & -1.352826464 & 17.622993068\end{array}$

H $\quad 20.433251165 \quad 1.307532575 \quad 19.614620836$

$\begin{array}{lllll}\mathrm{H} & 19.082791147 & 1.746486097 & 20.684473231\end{array}$

$\mathrm{H} \quad 19.047554692 \quad 2.233315619 \quad 18.972297541$

H $\quad 15.673895385 \quad-3.325735070 \quad 19.053558166$

H $\quad 16.537871287 \quad-2.542713942 \quad 20.394043286$

H $\quad 17.338792939-2.759543592 \quad 18.809500470$

$\begin{array}{llll}\mathrm{H} & 15.141212242 & -2.045073707 & 16.943072960\end{array}$

H $\quad 16.726884011 \quad-1.246839453 \quad 16.807426414$

$\mathrm{H} \quad 15.243230358 \quad-0.262599623 \quad 16.903595101$

H $\quad 13.724794971 \quad-1.815963354 \quad 18.896940653$

H $\quad 13.934222708-0.048309639 \quad 19.137211049$

H $\quad 14.315819583-1.185849435 \quad 20.453587545$

$\begin{array}{lllll}\mathrm{H} & 16.509740564 & 6.986210213 & 19.240909130\end{array}$

$\begin{array}{lllll}\mathrm{H} & 16.803022909 & 5.221883277 & 19.150486500\end{array}$

$\begin{array}{lllll}\mathrm{H} & 15.429143597 & 5.848781713 & 20.087649922\end{array}$

$\begin{array}{lllll}\mathrm{H} & 16.557817141 & 7.140948929 & 16.768805854\end{array}$

H $\quad 15.432969495 \quad 6.243009896 \quad 15.731320826$ 


$\begin{array}{llll}\mathrm{H} & 16.787783677 & 5.393545981 & 16.529964428 \\ \mathrm{H} & 11.892682754 & 4.642164148 & 14.964733585 \\ \mathrm{H} & 13.115256236 & 5.837885106 & 15.444898746 \\ \mathrm{H} & 11.839544695 & 5.346900097 & 16.596582792 \\ \mathrm{H} & 13.832118232 & 3.176528280 & 14.254149066 \\ \mathrm{H} & 15.013244517 & 2.574962437 & 15.450593778 \\ \mathrm{H} & 15.078636505 & 4.269890819 & 14.898242367 \\ \mathrm{H} & 12.056564091 & 2.253157877 & 15.616583174 \\ \mathrm{H} & 11.711259695 & 2.800942525 & 17.274961980 \\ \mathrm{H} & 13.121249181 & 1.750385647 & 16.964427287 \\ \mathrm{H} & 15.521995609 & 1.674293078 & 17.323413025 \\ \mathrm{C} & 17.251112946 & 2.944013933 & 17.349847273 \\ \mathrm{H} & 16.905396667 & 3.366064478 & 16.395139699 \\ \mathrm{H} & 17.936799850 & 2.123618354 & 17.098791266 \\ \mathrm{H} & 17.823502170 & 3.721614723 & 17.889769474\end{array}$

$==$
6

\begin{tabular}{|c|c|c|c|}
\hline $\mathrm{Rh}$ & 15.413834525 & 113643335 & 18.504535477 \\
\hline $\mathrm{P}$ & & & \\
\hline$P$ & 14.169964077 & 4.042784952 & \\
\hline $\mathrm{Si}$ & & & \\
\hline $\mathrm{Si}$ & & & \\
\hline $\mathrm{N}$ & & & \\
\hline $\mathrm{C}$ & & & \\
\hline & & & \\
\hline $\mathrm{H}$ & & & \\
\hline $\mathrm{H}$ & & & \\
\hline $\mathrm{C}$ & & & \\
\hline $\mathrm{H}$ & & & \\
\hline $\mathrm{H}$ & & & \\
\hline $\mathrm{H}$ & & & \\
\hline $\mathrm{C}$ & & & \\
\hline $\mathrm{H}$ & & & \\
\hline I & & & \\
\hline E & & & \\
\hline $\mathrm{C}$ & & & \\
\hline $\mathrm{C}$ & & & \\
\hline $\mathrm{H}$ & & & \\
\hline $\mathrm{H}$ & & & \\
\hline $\mathrm{H}$ & & & \\
\hline $\mathrm{C}$ & & & \\
\hline $\mathrm{H}$ & & & \\
\hline H & & & \\
\hline $\mathrm{H}$ & & & \\
\hline $\mathrm{C}$ & & & \\
\hline & & & \\
\hline & & & \\
\hline $\mathrm{C}$ & & & \\
\hline $\mathrm{C}$ & & & \\
\hline & & & \\
\hline & & & \\
\hline $\mathrm{H}$ & & & \\
\hline $\mathrm{C}$ & & & \\
\hline & & & \\
\hline & & & \\
\hline $\mathrm{C}$ & 14.340345926 & -1.2650150 & 19.870252065 \\
\hline $\mathrm{C}$ & & & \\
\hline & & & \\
\hline
\end{tabular}



$\begin{array}{llll}\text { C } & 18.647438682 & -0.483271403 & 17.474010227\end{array}$
$\begin{array}{llll}\text { C } & 12.203173305 & 5.117026398 & 16.021238574\end{array}$
$\begin{array}{llll}\text { C } & 12.758510288 & 2.675169461 & 15.920444025\end{array}$
$\begin{array}{lllll}\text { C } & 14.381143525 & 4.371409244 & 15.021400938\end{array}$
$\begin{array}{llll}\text { C } & 15.431271724 & 5.698200990 & 19.723640478\end{array}$
$\begin{array}{llll}\text { C } & 16.591779585 & 5.458900342 & 17.525419494\end{array}$
C $\quad 16.125323601 \quad 1.906220345 \quad 16.836605315$
H $\quad 17.229143839 \quad 6.323747112 \quad 17.785679897$
$\begin{array}{lllll}\mathrm{H} & 16.530263435 & 5.406703433 & 16.427706873\end{array}$
$\begin{array}{lllll}\mathrm{H} & 17.084545477 & 4.539960873 & 17.884366276\end{array}$
$\begin{array}{llll}\mathrm{H} & 20.122031486 & 1.484276258 & 18.692092393\end{array}$
$\mathrm{H} \quad 18.955478095 \quad 1.963417165 \quad 19.949831420$
$\begin{array}{lllll}\mathrm{H} & 18.533336004 & 2.176913563 & 18.234114109\end{array}$
$\begin{array}{llll}\mathrm{H} & 19.716668825 & -0.431098922 & 17.197612132\end{array}$
$\begin{array}{lllll}\mathrm{H} & 18.084570705 & 0.089203861 & 16.722786952\end{array}$
H $\quad 18.347986111 \quad-1.541515898 \quad 17.406372606$
H $\quad 20.338589460 \quad-0.789289484 \quad 19.582991956$
$\begin{array}{llll}\mathrm{H} & 18.945446244 & -1.851844730 & 19.879027375\end{array}$
H $\quad 19.225025799 \quad-0.433743567 \quad 20.919086188$
$\mathrm{H} \quad 14.673350145 \quad-2.368243756 \quad 17.402769823$
H $\quad 16.181611419-1.551208529 \quad 16.922403534$
H $\quad 14.722323798 \quad-0.583438455 \quad 17.258240702$
H $\quad 15.729688538$-3.515960416 19.440506868
$\begin{array}{lllll}\mathrm{H} & 16.744234544 & -2.586044378 & 20.565392685\end{array}$
$\begin{array}{lllll}\mathrm{H} & 17.283679373 & -2.860795670 & 18.882680178\end{array}$
$\begin{array}{lllll}\mathrm{H} & 13.670979334 & -2.095831551 & 19.581102768\end{array}$
H $\quad 13.826291563 \quad-0.310927403 \quad 19.667347346$
H $\quad 14.510246720 \quad-1.338939718 \quad 20.956404148$
$\begin{array}{lllll}\mathrm{H} & 16.172925946 & 6.488223427 & 19.942289013\end{array}$
$\mathrm{H} \quad 15.803698950 \quad 4.740627787 \quad 20.122448863$
$\begin{array}{llll}\mathrm{H} & 14.505421374 & 5.955074924 & 20.263432736\end{array}$
$\begin{array}{lllll}\mathrm{H} & 11.740925792 & 5.024880359 & 15.021375456\end{array}$
$\begin{array}{lllll}\mathrm{H} & 12.551364671 & 6.154224089 & 16.128248880\end{array}$
$\begin{array}{lllll}\mathrm{H} & 11.407592589 & 4.946384644 & 16.763179947\end{array}$
$\begin{array}{lllll}\mathrm{H} & 13.893614987 & 4.239521962 & 14.037919417\end{array}$
$\mathrm{H} \quad 15.239818184 \quad 3.685596427 \quad 15.057332215$
$\mathrm{H} \quad 14.755716475 \quad 5.406910606 \quad 15.059928562$
$\mathrm{H} \quad 12.248264246 \quad 2.636719544 \quad 14.940560585$
$\mathrm{H} \quad 12.013365994 \quad 2.417859920 \quad 16.692590535$
$\mathrm{H} \quad 13.540286152 \quad 1.900392908 \quad 15.938219005$
$\begin{array}{llll}\text { O } & 16.567429451 & 1.755690007 & 15.748948329\end{array}$

7

\begin{tabular}{cccc}
\hline $\mathrm{Rh}$ & 15.840895378 & 2.507552117 & 18.779918952 \\
$\mathrm{P}$ & 16.786751118 & 0.362157332 & 19.440063038 \\
$\mathrm{P}$ & 14.365266810 & 4.256412613 & 17.925106270 \\
$\mathrm{Si}$ & 14.933167306 & 1.255905529 & 21.852759918 \\
$\mathrm{Si}$ & 12.958750279 & 3.151805837 & 20.567091902 \\
$\mathrm{~N}$ & 14.401044914 & 2.233249069 & 20.552182718 \\
$\mathrm{C}$ & 15.646998618 & 2.203953101 & 23.345598453 \\
$\mathrm{H}$ & 14.879142583 & 2.820836769 & 23.825596078 \\
$\mathrm{H}$ & 16.462522286 & 2.872837849 & 23.048626496 \\
$\mathrm{H}$ & 16.038244136 & 1.517128119 & 24.107904636 \\
$\mathrm{C}$ & 13.608378416 & 0.104039395 & 22.597581521 \\
$\mathrm{H}$ & 12.834555724 & 0.685775613 & 23.113146534 \\
$\mathrm{H}$ & 14.045813552 & -0.574082587 & 23.341594520 \\
$\mathrm{H}$ & 13.104469135 & -0.504734153 & 21.840075425 \\
$\mathrm{C}$ & 16.401320842 & 0.169598387 & 21.237716917
\end{tabular}




\begin{tabular}{|c|c|c|c|}
\hline $\mathrm{H}$ & 17.284530973 & 0.522429829 & 21.782194752 \\
\hline $\mathrm{H}$ & 16.280546639 & -0.886910047 & 21.503311269 \\
\hline $\mathrm{C}$ & 15.909346547 & -1.124640027 & 18.590706844 \\
\hline $\mathrm{C}$ & 18.709038116 & 0.104401604 & 19.385797620 \\
\hline $\mathrm{C}$ & 12.600518279 & 4.083274856 & 22.196864621 \\
\hline $\mathrm{H}$ & 13.439381757 & 4.727487577 & 22.486280449 \\
\hline $\mathrm{H}$ & 12.414551932 & 3.394426044 & 23.029449813 \\
\hline $\mathrm{H}$ & 11.709428079 & 4.717974482 & 22.101937754 \\
\hline $\mathrm{C}$ & 11.364933650 & 2.156237790 & 20.245513021 \\
\hline $\mathrm{H}$ & 11.462873753 & 1.448988017 & 19.417433473 \\
\hline $\mathrm{H}$ & 10.501375377 & 2.803191588 & 20.043076517 \\
\hline $\mathrm{H}$ & 11.123723707 & 1.571489571 & 21.141586438 \\
\hline $\mathrm{C}$ & 13.163950250 & 4.577932360 & 6975 \\
\hline $\mathrm{H}$ & 12.212834214 & 4.968164959 & 18.911162799 \\
\hline $\mathrm{H}$ & 13.609421487 & 5.383676383 & 19.888145450 \\
\hline $\mathrm{C}$ & 15.082103037 & 6.030531216 & 5913 \\
\hline $\mathrm{C}$ & 14.017142529 & 7.141331237 & 18671 \\
\hline $\mathrm{H}$ & 14.494775493 & 8.109156462 & 4393 \\
\hline $\mathrm{H}$ & 13.606083212 & 7.188617407 & 36092 \\
\hline $\mathrm{H}$ & 13.185594596 & 7.039046022 & 39778 \\
\hline $\mathrm{C}$ & 13.279272819 & 3.708150727 & 6042 \\
\hline $\mathrm{C}$ & 16.499877783 & -2.496250901 & 97750 \\
\hline $\mathrm{C}$ & 15.948845831 & -0.976094113 & 1184 \\
\hline $\mathrm{C}$ & 14.433734134 & -1.093612195 & 80088 \\
\hline $\mathrm{C}$ & 19.210087716 & -0.186138285 & 8434 \\
\hline $\mathrm{C}$ & 19.195582818 & -1.023931085 & 4908 \\
\hline $\mathrm{C}$ & 19.360346708 & 1.416433929 & 1613 \\
\hline $\mathrm{C}$ & 12.373599792 & 4.82988 & 3784 \\
\hline $\mathrm{C}$ & 12.356381350 & 2.568339634 & 1020 \\
\hline $\mathrm{C}$ & 14.168575881 & 3.162791798 & 7666 \\
\hline $\mathrm{C}$ & 16.190153840 & 0260 & 407 \\
\hline $\mathrm{C}$ & 15.715551511 & 6.139812038 & 6236 \\
\hline $\mathrm{H}$ & & & \\
\hline $\mathrm{H}$ & 18.824694285 & -2.009003702 & 2406 \\
\hline $\mathrm{H}$ & 18.921296259 & -0.846121140 & 860228 \\
\hline $\mathrm{H}$ & 20.307672612 & -0.172279975 & 5174 \\
\hline $\mathrm{H}$ & 18.868484535 & 0.561508261 & 4348 \\
\hline $\mathrm{H}$ & 18.902210702 & -1.174148509 & 17.604229126 \\
\hline $\mathrm{H}$ & 20.448346954 & 1.280472833 & 6205 \\
\hline $\mathrm{H}$ & 19.035983834 & 1.695636782 & 20.880226955 \\
\hline $\mathrm{H}$ & 19.150875297 & 2.251590294 & 19.202977673 \\
\hline $\mathrm{H}$ & 15.840829108 & -3.283340305 & 46517 \\
\hline $\mathrm{H}$ & 16.566569346 & -2.637326515 & 20.058917964 \\
\hline $\mathrm{H}$ & 17.489035651 & -2.663901650 & 2123384 \\
\hline $\mathrm{H}$ & 15.397006342 & -1.810468277 & 16.605450128 \\
\hline $\mathrm{H}$ & 16.964134816 & -0.999370137 & 16.653310936 \\
\hline $\mathrm{H}$ & 15.475032645 & -0.044948771 & 16.738558614 \\
\hline $\mathrm{H}$ & 13.861321666 & -1.808232519 & 18.442139484 \\
\hline $\mathrm{H}$ & 13.988572447 & -0.102741606 & 18.930973572 \\
\hline $\mathrm{H}$ & 14.327993762 & -1.391942055 & 20.093519029 \\
\hline $\mathrm{H}$ & 16.536903749 & 7.342197546 & 18.469145019 \\
\hline $\mathrm{H}$ & 17.049217129 & 5.650274097 & 18.436967463 \\
\hline $\mathrm{H}$ & 15.841506369 & 6.195219642 & 19.615378176 \\
\hline $\mathrm{H}$ & 16.195784693 & 7.122599351 & 16.054579447 \\
\hline $\mathrm{H}$ & 14.981309894 & 6.066061498 & 15.341633634 \\
\hline $\mathrm{H}$ & 16.487907218 & 5.385453242 & 15.980173464 \\
\hline $\mathrm{H}$ & 11.702800002 & 4.400176437 & 15.125092317 \\
\hline $\mathrm{H}$ & 12.933658033 & 5.629832786 & 15.391422722 \\
\hline $\mathrm{H}$ & 11.741191217 & 5.272440124 & 16.655624221 \\
\hline
\end{tabular}




$\begin{array}{cccc}\mathrm{H} & 13.526528425 & 2.807406431 & 14.478011237 \\ \mathrm{H} & 14.774815674 & 2.323483252 & 15.640036156 \\ \mathrm{H} & 14.838805848 & 3.917825658 & 14.877378271 \\ \mathrm{H} & 11.821012099 & 2.164039599 & 16.035882773 \\ \mathrm{H} & 11.607975039 & 2.918120091 & 17.618234651 \\ \mathrm{H} & 12.918532108 & 1.751968432 & 17.363216065 \\ \mathrm{H} & 14.934797416 & 1.619627108 & 17.842772251 \\ \mathrm{C} & 17.145986295 & 2.661322616 & 17.098732323 \\ \mathrm{H} & 16.881228556 & 3.501131367 & 16.455630495 \\ \mathrm{H} & 17.098879378 & 1.754959847 & 16.490658505 \\ \mathrm{H} & 18.178495373 & 2.810559753 & 17.426834004 \\ \mathrm{C} & 16.950599470 & 3.598558006 & 20.010926868 \\ \mathrm{O} & 17.537225637 & 4.192051811 & 20.803854123\end{array}$

8

\begin{tabular}{cccc}
\hline $\mathrm{Rh}$ & 15.673770579 & 2.349757390 & 18.604268151 \\
$\mathrm{P}$ & 16.818958006 & 0.310381770 & 19.445332961 \\
$\mathrm{P}$ & 14.392254171 & 4.364198030 & 17.893463159 \\
$\mathrm{Si}$ & 14.865696491 & 1.277556784 & 21.801402127 \\
$\mathrm{Si}$ & 12.827104726 & 3.057463959 & 20.378681346 \\
$\mathrm{~N}$ & 14.218695256 & 2.067979870 & 20.429476865 \\
$\mathrm{C}$ & 15.375427822 & 2.372657641 & 23.282269707 \\
$\mathrm{H}$ & 14.501701839 & 2.840493616 & 23.748846712 \\
$\mathrm{H}$ & 16.066144247 & 3.172031573 & 22.996803466 \\
$\mathrm{H}$ & 15.867943939 & 1.767277590 & 24.055165358 \\
$\mathrm{C}$ & 13.704019619 & -0.004032742 & 22.609690405 \\
$\mathrm{H}$ & 12.956746408 & 0.525870091 & 23.213490139 \\
$\mathrm{H}$ & 14.240793009 & -0.675050211 & 23.292569501 \\
$\mathrm{H}$ & 13.159367598 & -0.616936196 & 21.887061944 \\
$\mathrm{C}$ & 16.545698186 & 0.455301847 & 21.272792128 \\
$\mathrm{H}$ & 17.323785882 & 1.133956913 & 21.642354842 \\
$\mathrm{H}$ & 16.714586206 & -0.500457887 & 21.780540444 \\
$\mathrm{C}$ & 16.163835711 & -1.486866834 & 19.080457649 \\
$\mathrm{C}$ & 18.750716290 & 0.310535123 & 19.213239532 \\
$\mathrm{C}$ & 12.280484533 & 3.826358102 & 22.041359772 \\
$\mathrm{H}$ & 13.064512369 & 4.446423521 & 22.490629267 \\
$\mathrm{H}$ & 12.011563919 & 3.055590423 & 22.773578668 \\
$\mathrm{H}$ & 11.394222890 & 4.459583864 & 21.904757781 \\
$\mathrm{C}$ & 11.267614804 & 2.156976734 & 19.764426551 \\
$\mathrm{H}$ & 11.446744200 & 1.545100138 & 18.876743069 \\
$\mathrm{H}$ & 10.433824416 & 2.837873593 & 19.550968154 \\
$\mathrm{H}$ & 10.934757803 & 1.480119433 & 20.561557874 \\
$\mathrm{C}$ & 13.243135363 & 4.627196342 & 19.320372288 \\
$\mathrm{H}$ & 12.359384343 & 5.204558783 & 19.024807923 \\
$\mathrm{H}$ & 13.795687030 & 5.255953584 & 20.030726767 \\
$\mathrm{C}$ & 15.375415961 & 6.037985942 & 17.732668304 \\
$\mathrm{C}$ & 14.487096062 & 7.274152492 & 18.002246138 \\
$\mathrm{H}$ & 15.107604181 & 8.174442720 & 17.910341813 \\
$\mathrm{H}$ & 14.081962217 & 7.265229033 & 19.017857261 \\
$\mathrm{H}$ & 13.655082900 & 7.382113588 & 17.310147273 \\
$\mathrm{C}$ & 13.242998174 & 4.265903180 & 16.335934462 \\
$\mathrm{C}$ & 17.015955806 & -2.565848897 & 19.786835787 \\
$\mathrm{C}$ & 16.100300928 & -1.861337638 & 17.581514837 \\
$\mathrm{C}$ & 14.736435115 & -1.573079420 & 19.662519192 \\
& 19.124856162 & -0.263905801 & 17.832711159 \\
$\mathrm{C}$ & 19.500435028 & -0.457030792 & 20.323907153 \\
$\mathrm{C}$ & 12.521632096 & 5.586508557 & 15.985152007
\end{tabular}




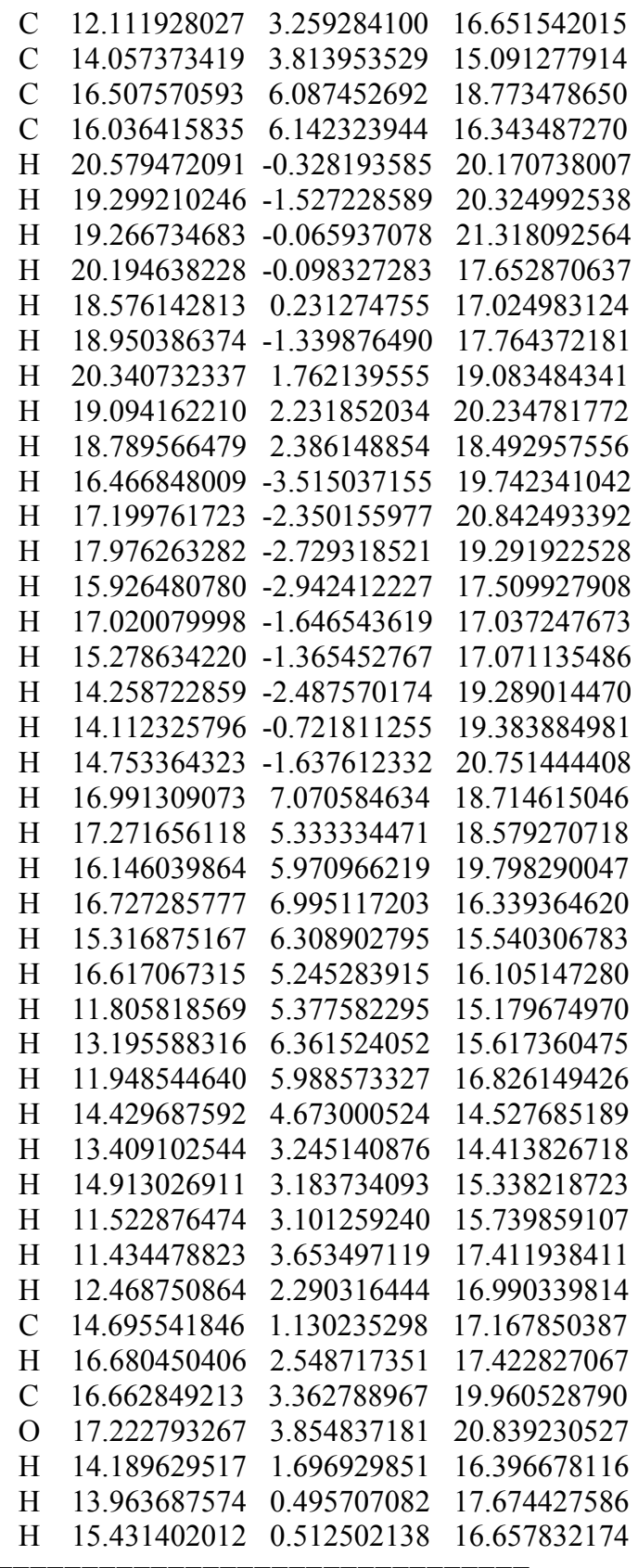

9

\begin{tabular}{|c|c|c|c|}
\hline $\mathrm{Rh}$ & 14.763169337 & 2.145480362 & 18.556664504 \\
\hline $\mathrm{P}$ & 16.310486348 & 0.364979929 & 19.079948932 \\
\hline $\mathrm{P}$ & 14.017382333 & 4.241047549 & 18.099708617 \\
\hline $\mathrm{Si}$ & 15.313611739 & 1.629749185 & 21.866513507 \\
\hline $\mathrm{Si}$ & 13.106586020 & 3.665516383 & 21.004420080 \\
\hline $\mathrm{N}$ & 14.439136361 & 2.597131312 & 20.740149582 \\
\hline $\mathrm{C}$ & 16.643317461 & 2.589322925 & 22.846742742 \\
\hline $\mathrm{H}$ & 16.141177781 & 3.257249307 & 23.569376678 \\
\hline $\mathrm{H}$ & 17.271500137 & 3.222306527 & 22.200620446 \\
\hline $\mathrm{H}$ & 17.305097839 & 1.920320293 & 23.426106443 \\
\hline $\mathrm{C}$ & 14.227449419 & 0.803305073 & 23.206429086 \\
\hline $\mathrm{H}$ & 13.742081067 & 1.558448207 & 23.849779380 \\
\hline
\end{tabular}


$\begin{array}{llll}\mathrm{H} & 14.824023230 & 0.147961594 & 23.866617029\end{array}$

$\begin{array}{llll}\mathrm{H} & 13.428048708 & 0.192934880 & 22.752032359\end{array}$

$\begin{array}{llll}\text { C } & 16.094607365 & 0.128428112 & 20.913267762\end{array}$

$\begin{array}{llll}\mathrm{H} & 17.017295439 & -0.251251748 & 21.390513815\end{array}$

$\begin{array}{llll}\mathrm{H} & 15.340152608 & -0.674605129 & 21.017493183\end{array}$

$\begin{array}{llll}\text { C } & 16.059383163 & -1.412680546 & 18.345412811\end{array}$

$\begin{array}{llll}\text { C } & 18.165481160 & 0.865328878 & 18.862853166\end{array}$

$\begin{array}{llll}\text { C } & 13.403811987 & 4.946456358 & 22.389809528\end{array}$

$\begin{array}{lllll}\mathrm{H} & 14.371438938 & 5.464473279 & 22.300430259\end{array}$

$\begin{array}{lllll}\mathrm{H} & 13.398563494 & 4.429017900 & 23.366185127\end{array}$

$\begin{array}{lllll}\mathrm{H} & 12.602760937 & 5.706387205 & 22.424633959\end{array}$

C $\quad 11.454091642 \quad 2.832434724 \quad 21.475540283$

$\begin{array}{lllll}\mathrm{H} & 11.134803243 & 2.092504530 & 20.724044681\end{array}$

$\begin{array}{lllll}\mathrm{H} & 10.647975859 & 3.580611307 & 21.585680193\end{array}$

$\begin{array}{llll}\mathrm{H} & 11.550784522 & 2.304218444 & 22.439733870\end{array}$

$\begin{array}{llll}\text { C } & 12.680535915 & 4.598709984 & 19.314937142\end{array}$

$\begin{array}{lllll}\mathrm{H} & 11.761332577 & 4.121186235 & 18.930369347\end{array}$

$\begin{array}{llll}\mathrm{H} & 12.466889711 & 5.675446208 & 19.438261560\end{array}$

$\begin{array}{lllll}\text { C } & 15.196881103 & 5.737490804 & 18.167350328\end{array}$

$\begin{array}{llll}\text { C } & 14.475947490 & 6.985609293 & 17.614720485\end{array}$

$\begin{array}{lllll}\mathrm{H} & 15.114188977 & 7.873684641 & 17.773357133\end{array}$

$\begin{array}{lllll}\mathrm{H} & 13.520232770 & 7.174065043 & 18.131555750\end{array}$

$\begin{array}{lllll}\mathrm{H} & 14.274355666 & 6.907038806 & 16.535498748\end{array}$

$\begin{array}{lllll}\text { C } & 13.615346227 & 3.657859188 & 16.313790695\end{array}$

$\begin{array}{llll}\text { C } & 16.711717995 & -2.531235532 & 19.184496894\end{array}$

$\begin{array}{llll}\text { C } & 16.566730824 & -1.464816498 & 16.891130578\end{array}$

C $\quad 14.539132561 \quad-1.666528309 \quad 18.318083195$

$\begin{array}{llll}\text { C } & 19.165181979 & -0.274922502 & 19.139979481\end{array}$

$\begin{array}{llll}\text { C } & 18.460266840 & 1.992736085 & 19.873273782\end{array}$

$\begin{array}{llll}\text { C } & 18.382100623 & 1.416979541 & 17.438500113\end{array}$

$\begin{array}{llll}\text { C } & 13.859823103 & 4.599056302 & 15.124306567\end{array}$

$\begin{array}{llll}\text { C } & 12.149092314 & 3.198504228 & 16.250059330\end{array}$

$\begin{array}{llll}\text { C } & 14.617813732 & 2.459708272 & 16.407461493\end{array}$

$\begin{array}{llll}\text { C } & 15.553962236 & 5.959001773 & 19.652437365\end{array}$

$\begin{array}{llll}\text { C } & 16.498801034 & 5.487064891 & 17.386328179\end{array}$

$\begin{array}{llll}\mathrm{H} & 19.469775762 & 2.397467048 & 19.673551289\end{array}$

$\begin{array}{lllll}\mathrm{H} & 18.445843821 & 1.629444901 & 20.912664187\end{array}$

$\begin{array}{lllll}\mathrm{H} & 17.736528693 & 2.820013487 & 19.785506139\end{array}$

$\begin{array}{lllll}\mathrm{H} & 20.190135186 & 0.140343658 & 19.130826131\end{array}$

$\begin{array}{lllll}\mathrm{H} & 19.132196925 & -1.0680386886 & 18.377051686\end{array}$

H $\quad 19.010977051 \quad-0.733808606 \quad 20.131227615$

$\begin{array}{llll}\mathrm{H} & 19.409887074 & 1.816401088 & 17.356156766\end{array}$

$\begin{array}{lllll}\mathrm{H} & 17.673162828 & 2.233253854 & 17.225723238\end{array}$

$\begin{array}{lllll}\mathrm{H} & 18.260473097 & 0.647902067 & 16.660542973\end{array}$

$\begin{array}{lllll}\mathrm{H} & 16.453256408 & -3.508915002 & 18.737025478\end{array}$

H $\quad 16.337110485 \quad-2.538769974 \quad 20.220861898$

H $\quad 17.807509620 \quad-2.465571878 \quad 19.219601446$

H $\quad 16.231863271 \quad-2.408313079 \quad 16.421357094$

$\mathrm{H} \quad 17.665737875 \quad-1.440235756 \quad 16.822943263$

$\mathrm{H} \quad 16.160729004 \quad-0.630708958 \quad 16.293489701$

$\mathrm{H} \quad 14.352301692 \quad-2.704517560 \quad 17.986631204$

H $\quad 14.033857099-0.988540156 \quad 17.614478010$

$\begin{array}{lllll}\mathrm{H} & 14.068096724 & -1.546569918 & 19.308196903\end{array}$

$\mathrm{H} \quad 16.324753552 \quad 6.744813468 \quad 19.723991201$

H $\quad 15.943083651 \quad 5.039643356 \quad 20.116947472$

$\begin{array}{lllll}\mathrm{H} & 14.685350958 & 6.294745897 & 20.236032220\end{array}$

$\begin{array}{lllll}\mathrm{H} & 17.146883163 & 6.376421102 & 17.483995908\end{array}$

$\begin{array}{lllll}\mathrm{H} & 16.335317996 & 5.314038738 & 16.312216407\end{array}$

$\begin{array}{lllll}\mathrm{H} & 17.041545643 & 4.619941378 & 17.793494922\end{array}$ 


$\begin{array}{cccc}\mathrm{H} & 13.678637745 & 4.035966259 & 14.189582294 \\ \mathrm{H} & 14.890221307 & 4.980512352 & 15.075965152 \\ \mathrm{H} & 13.168226091 & 5.460139057 & 15.128510045 \\ \mathrm{H} & 15.599209714 & 2.733019805 & 15.989183735 \\ \mathrm{H} & 11.988765411 & 2.642070780 & 15.308612668 \\ \mathrm{H} & 11.457338856 & 4.058402611 & 16.252962204 \\ \mathrm{H} & 11.870250004 & 2.530041504 & 17.076643107 \\ \mathrm{H} & 14.234762655 & 1.559731493 & 15.898055538 \\ \mathrm{H} & 16.142127969 & 2.975050036 & 18.592727045 \\ \mathrm{C} & 13.110685330 & 1.159734083 & 18.649895394 \\ \mathrm{O} & 12.112594945 & 0.578771980 & 18.839986858\end{array}$

10

$\begin{array}{llll}\mathrm{Rh} & 15.391000664 & 2.554962048 & 18.722823487\end{array}$

$\begin{array}{llll}\text { P } & 16.435502842 & 0.399039548 & 19.169109030\end{array}$

$\begin{array}{llll}\text { P } & 13.980918299 & 4.325772072 & 18.197115480\end{array}$

Si $\quad \begin{array}{llll}15.282983650 & 1.551602724 & 21.885721388\end{array}$

Si $\quad 12.822100479 \quad 3.129165230 \quad 20.871865396$

$\begin{array}{llll}\mathrm{N} & 14.287177285 & 2.251630252 & 20.664495206\end{array}$

C $\quad 16.277498116 \quad 2.796141326 \quad 22.942288761$

$\begin{array}{lllll}\mathrm{H} & 15.596677223 & 3.426135050 & 23.539812485\end{array}$

$\mathrm{H} \quad 16.891319688 \quad 3.468639700 \quad 22.321062931$

$\begin{array}{lllll}\mathrm{H} & 16.952122796 & 2.278675343 & 23.648028001\end{array}$

C $\quad 14.344625589 \quad 0.477172326 \quad 23.158200855$

$\begin{array}{lllll}\mathrm{H} & 13.700554256 & 1.110842360 & 23.792514797\end{array}$

H $\quad 15.045697091 \quad-0.045332902 \quad 23.832645786$

$\begin{array}{lllll}\mathrm{H} & 13.697231729 & -0.279043839 & 22.685873357\end{array}$

$\begin{array}{llll}\text { C } & 16.626941701 & 0.454138616 & 21.012723173\end{array}$

$\begin{array}{lllll}\mathrm{H} & 17.596898211 & 0.948846115 & 21.204152488\end{array}$

$\begin{array}{llll}\mathrm{H} & 16.699859576 & -0.558270716 & 21.448296482\end{array}$

$\begin{array}{llll}\text { C } & 15.425330277 & -1.219428753 & 18.874480162\end{array}$

$\begin{array}{llll}\text { C } & 18.238955347 & 0.156844292 & 18.504074314\end{array}$

C $\quad 12.622709490 \quad 3.990763196 \quad 22.567007286$

$\begin{array}{lllll}\mathrm{H} & 13.459578712 & 4.683595706 & 22.759939272\end{array}$

$\begin{array}{lllll}\mathrm{H} & 12.597951209 & 3.262836453 & 23.396085492\end{array}$

$\begin{array}{lllll}\mathrm{H} & 11.683902119 & 4.570096054 & 22.614920840\end{array}$

C $\quad 11.230150653 \quad 2.101294575 \quad 20.640984192$

$\begin{array}{llll}\mathrm{H} & 11.189439715 & 1.587876820 & 19.667100122\end{array}$

$\begin{array}{lllll}\mathrm{H} & 10.322531405 & 2.723393982 & 20.736257233\end{array}$

$\begin{array}{llll}\mathrm{H} & 11.179793695 & 1.321474657 & 21.420005728\end{array}$

$\begin{array}{llll}\text { C } & 12.818078109 & 4.594675961 & 19.600914312\end{array}$

$\begin{array}{lllll}\mathrm{H} & 11.813534227 & 4.881272602 & 19.243506505\end{array}$

$\begin{array}{lllll}\mathrm{H} & 13.214959490 & 5.449942211 & 20.174860787\end{array}$

$\begin{array}{llll}\text { C } & 14.543561108 & 6.057651890 & 17.608866473\end{array}$

$\begin{array}{llll}\text { C } & 13.342480755 & 6.805401918 & 16.996667483\end{array}$

$\begin{array}{lllll}\mathrm{H} & 13.650159446 & 7.827707269 & 16.710602499\end{array}$

$\begin{array}{lllll}\mathrm{H} & 12.511683130 & 6.899516858 & 17.715231975\end{array}$

$\begin{array}{lllll}\mathrm{H} & 12.959932410 & 6.307890434 & 16.091874773\end{array}$

$\begin{array}{lllll}\text { C } & 13.097727102 & 3.252343957 & 16.926728557\end{array}$

$\begin{array}{llll}\text { C } & 16.182512910 & -2.501894478 & 19.277020126\end{array}$

$\begin{array}{llll}\text { C } & 14.979836637 & -1.353918416 & 17.403991954\end{array}$

$\begin{array}{llll}\text { C } & 14.166417260 & -1.103017528 & 19.762426729\end{array}$

$\begin{array}{llll}\text { C } & 19.069797685 & -0.830127543 & 19.352578609\end{array}$

$\begin{array}{llll}\text { C } & 18.944112380 & 1.527949802 & 18.561336859\end{array}$

$\begin{array}{llll}\text { C } & 18.214550258 & -0.298579304 & 17.031314999\end{array}$

$\begin{array}{llll}\text { C } & 13.669084193 & 3.401610810 & 15.508670715\end{array}$

$\begin{array}{llll}\text { C } & 11.567737312 & 3.355873720 & 16.882899049\end{array}$

$\begin{array}{llll}\text { C } & 13.646449028 & 1.945998452 & 17.573589788\end{array}$ 

$\begin{array}{llll}\text { C } & 15.071985974 & 6.843378478 & 18.828529374\end{array}$
$\begin{array}{lllll}\text { C } & 15.690470289 & 5.925088469 & 16.588288828\end{array}$
H $\quad 19.987415751 \quad 1.404560800 \quad 18.218818687$
$\begin{array}{lllll}\mathrm{H} & 18.982966907 & 1.940502886 & 19.582755837\end{array}$
$\begin{array}{lllll}\mathrm{H} & 18.450405341 & 2.263166760 & 17.908891101\end{array}$
$\begin{array}{llll}\mathrm{H} & 20.101323220 & -0.851576611 & 18.961147612\end{array}$
H $\quad 18.686589773 \quad-1.858018464 \quad 19.324137444$
H $\quad 19.128192939-0.516496430 \quad 20.405968923$
$\begin{array}{lllll}\mathrm{H} & 19.236383924 & -0.233597056 & 16.614062687\end{array}$
$\begin{array}{lllll}\mathrm{H} & 17.562518418 & 0.346531666 & 16.417517345\end{array}$
H $\quad 17.883977437 \quad-1.344341507 \quad 16.919790427$
H $\quad 15.466447236-3.344226862 \quad 19.279412361$
$\begin{array}{lllll}\mathrm{H} & 16.617429262 & -2.443389655 & 20.288047037\end{array}$
$\begin{array}{lllll}\mathrm{H} & 16.982436812 & -2.759381433 & 18.565233489\end{array}$
$\begin{array}{lllll}\mathrm{H} & 14.407582177 & -2.294409961 & 17.296146595\end{array}$
H $\quad 15.824144278-1.397538451 \quad 16.699709332$
H $\quad 14.320923701-0.528237250 \quad 17.101071372$
H $\quad 13.443710298 \quad-1.884647966 \quad 19.465387446$
H $\quad 13.682999364-0.116879723 \quad 19.679626280$
H $\quad 14.411146069-1.265582385 \quad 20.823268205$
$\begin{array}{llll}\mathrm{H} & 15.489273209 & 7.804093535 & 18.477007391\end{array}$
$\mathrm{H} \quad 15.875416512 \quad 6.304781111 \quad 19.354502611$
$\begin{array}{lllll}\mathrm{H} & 14.278123427 & 7.082450694 & 19.552830468\end{array}$
$\mathrm{H} \quad 16.070571652 \quad 6.932708636 \quad 16.339566535$
$\begin{array}{lllll}\mathrm{H} & 15.368673306 & 5.449498421 & 15.651482314\end{array}$
$\begin{array}{lllll}\mathrm{H} & 16.525244833 & 5.337137367 & 17.001382938\end{array}$
$\begin{array}{llll}\mathrm{H} & 13.251456858 & 2.593486658 & 14.881098663\end{array}$
H $\quad 14.765672342 \quad 3.294193382 \quad 15.502535106$
$\begin{array}{lllll}\mathrm{H} & 13.396988247 & 4.360471867 & 15.035531867\end{array}$
$\begin{array}{llll}\mathrm{H} & 13.898445309 & 1.214643090 & 16.792340306\end{array}$
$\begin{array}{llll}\mathrm{H} & 11.170696761 & 2.560951289 & 16.225464922\end{array}$
$\begin{array}{lllll}\mathrm{H} & 11.228754124 & 4.325300037 & 16.475213771\end{array}$
$\mathrm{H} \quad 11.111757473 \quad 3.218884673 \quad 17.875128319$
$\begin{array}{lllll}\mathrm{H} & 12.929510061 & 1.503946870 & 18.282396024\end{array}$
$\begin{array}{lllll}\mathrm{H} & 16.059837677 & 2.636650307 & 17.280357165\end{array}$
C $\quad 16.808031866 \quad 3.590863364 \quad 19.479073241$
$\begin{array}{lllll}\text { O } & 17.593556562 & 4.397445704 & 19.804345904\end{array}$

\begin{tabular}{|c|c|c|c|}
\hline \multicolumn{4}{|l|}{11} \\
\hline $\mathrm{Rh}$ & 15.318141209 & 2.427577566 & 18.600397717 \\
\hline $\mathrm{P}$ & 16.487903752 & 0.404549745 & 19.228271118 \\
\hline $\mathrm{P}$ & 13.947496550 & 4.289957168 & 18.178635646 \\
\hline $\mathrm{Si}$ & 15.327407174 & 1.692219626 & 21.818418943 \\
\hline $\mathrm{Si}$ & 12.82 & 3.10 & 0283872 \\
\hline $\mathrm{N}$ & 14.302259581 & 2.242856187 & 20.532944267 \\
\hline $\mathrm{C}$ & 16.374795241 & 3.047863906 & 019118 \\
\hline $\mathrm{H}$ & & & 8563892 \\
\hline $\mathrm{H}$ & 16.93 & 3.61 & 268080 \\
\hline $\mathrm{H}$ & 17.100 & 2.622710522 & 23.373775060 \\
\hline $\mathrm{C}$ & 14.405 & 0.7402151 & 23.196068413 \\
\hline $\mathrm{H}$ & 13.73 & 1.38 & \\
\hline $\mathrm{H}$ & 15.129196354 & 0.289781920 & 23.898186994 \\
\hline $\mathrm{H}$ & 13.789844738 & -0.074704337 & 22.778164448 \\
\hline $\mathrm{C}$ & 16.596500169 & 0.458463481 & 21.070178200 \\
\hline $\mathrm{H}$ & 17.601499241 & 0.834506518 & \\
\hline $\mathrm{H}$ & 16.512426152 & -0.554589017 & 21.500074990 \\
\hline $\mathrm{C}$ & 15.530816333 & -1.217567303 & 18.839906135 \\
\hline & 18.342352870 & $0.25000404 \mathrm{~J}$ & \\
\hline
\end{tabular}


C $\quad 12.660353588 \quad 3.920463814 \quad 22.543513441$

$\begin{array}{llll}\mathrm{H} & 13.522580927 & 4.568104845 & 22.774601138\end{array}$

H $\quad 12.561273661 \quad 3.187488582 \quad 23.360644891$

H $\quad 11.753520829 \quad 4.550947590 \quad 22.564240830$

C $\quad 11.224327559 \quad 2.090692126 \quad 20.628720795$

H $\quad 11.125064202 \quad 1.587810101 \quad 19.654005339$

H $\quad 10.332822516 \quad 2.725831662 \quad 20.780095910$

H $\quad 11.202897886 \quad 1.305875360 \quad 21.405358240$

C $\quad 12.855282014 \quad 4.609074043 \quad 19.622410053$

H $\quad 11.867045995 \quad 4.975623168 \quad 19.297853435$

H $\quad 13.328899309 \quad 5.412930444 \quad 20.211501340$

C $\quad \begin{array}{llll}14.597681167 & 6.014927808 & 17.655587231\end{array}$

$\begin{array}{llll}\text { C } & 13.410684887 & 6.929774052 & 17.296971149\end{array}$

H $\quad 13.782217180 \quad 7.940631790 \quad 17.046987989$

H $\quad \begin{array}{llll}12.702900695 & 7.034374167 & 18.135637904\end{array}$

$\begin{array}{lllll}\mathrm{H} & 12.853991787 & 6.554966848 & 16.421489258\end{array}$

$\begin{array}{llll}\text { C } & 12.907670535 & 3.291686247 & 16.948692512\end{array}$

$\begin{array}{llll}\text { C } & 16.253091909 & -2.512713733 & 19.255531321\end{array}$

C $\quad 15.202129811 \quad-1.267940729 \quad 17.334550345$

C $\quad 14.208729257-1.106285483 \quad 19.633264472$

$\begin{array}{llll}\text { C } & 19.120172064 & -0.750239016 & 19.594129231\end{array}$

C $\quad 18.997654886 \quad 1.628747109 \quad 18.856477410$

$\begin{array}{llll}\text { C } & 18.475645714 & -0.216072737 & 17.228646151\end{array}$

C $\quad 13.355866652 \quad 3.479976328 \quad 15.490050796$

C $\quad 11.388104339 \quad 3.510597687 \quad 17.027855219$

$\begin{array}{llll}\text { C } & 13.393309000 & 1.917430412 & 17.504126159\end{array}$

C $\quad \begin{array}{llll}15.377145875 & 6.576735012 & 18.864233198\end{array}$

C $\quad 15.573547622 \quad 5.907485448 \quad 16.469324217$

$\begin{array}{lllll}\mathrm{H} & 20.079303283 & 1.532913007 & 18.648993970\end{array}$

$\begin{array}{llll}\mathrm{H} & 18.885919466 & 2.028156873 & 19.878020493\end{array}$

H $\quad \begin{array}{llll}18.578739597 & 2.372301641 & 18.165068746\end{array}$

H $20.173419515 \quad-0.767634311 \quad 19.259749419$

H $\quad 18.739007703 \quad-1.778698078 \quad 19.533565335$

H $19.120531992 \quad-0.447436240 \quad 20.652704832$

H $19.534237212-0.130291913 \quad 16.922009132$

$\begin{array}{llll}\mathrm{H} & 17.883040491 & 0.399380466 & 16.536013316\end{array}$

H $\quad 18.185770729-1.270561959 \quad 17.096620838$

H $\quad 15.549118971 \quad-3.359153131 \quad 19.150815839$

H $\quad 16.585793866 \quad-2.495656860 \quad 20.306843633$

$\begin{array}{llll}\mathrm{H} & 17.122657600 & -2.734083725 & 18.616278807\end{array}$

H $\quad 14.517617783 \quad-2.114119175 \quad 17.142832865$

H $16.094985266-1.416453382 \quad 16.708834993$

H $\quad 14.703845666 \quad-0.342568361 \quad 17.011983047$

H $\quad 13.514266267 \quad-1.892433767 \quad 19.284646489$

H $13.732634231-0.121712568 \quad 19.500771585$

H $\quad 14.368430882 \quad-1.259729278 \quad 20.713060480$

H $\quad 15.887134441 \quad 7.509216198 \quad 18.562766004$

H $\quad 16.143715332 \quad 5.862459280 \quad 19.211428450$

$\begin{array}{llll}\mathrm{H} & 14.722415397 & 6.823470113 & 19.715463346\end{array}$

H $\quad 15.972582754 \quad 6.913425890 \quad 16.243860271$

H $\quad 15.094718081 \quad 5.528734715 \quad 15.554860861$

H $\quad 16.429294475 \quad 5.257603317 \quad 16.708890339$

H $\quad 12.843601209 \quad 2.722063927 \quad 14.869402192$

H $\quad 14.437144374 \quad 3.335055496 \quad 15.352372208$

H $\quad 13.079843785 \quad 4.472257789 \quad 15.093325527$

H $\quad 13.495901903 \quad 1.190083626 \quad 16.681760910$

$\begin{array}{llll}\mathrm{H} & 10.888618071 & 2.788498568 & 16.356482961\end{array}$

H $\quad 11.092522011 \quad 4.525562856 \quad 16.703717381$

H $\quad 10.993439776 \quad 3.340696727 \quad 18.040823895$ 
H $\quad 12.700831472 \quad 1.511479439 \quad 18.255612007$

C $\quad 16.247210543 \quad 2.568618541 \quad 17.017314364$

$\begin{array}{lllll}\mathrm{H} & 16.525649154 & 3.140421437 & 19.446946450\end{array}$

O $\quad 16.878770524 \quad 2.658146329 \quad 16.029413078$ 